\title{
The fourth flight of CHESS: spectral resolution enhancements for high- resolution FUV spectroscopy
}

Nicholas Kruczek, Nicholas Nell, Kevin France, Keri Hoadley, Brian Fleming, et al.

Nicholas Kruczek, Nicholas Nell, Kevin France, Keri Hoadley, Brian Fleming, Stefan Ulrich, Alexander D. Miller, Arika Egan, Emily Witt, Robert Kane, "The fourth flight of CHESS: spectral resolution enhancements for highresolution FUV spectroscopy," Proc. SPIE 10699, Space Telescopes and Instrumentation 2018: Ultraviolet to Gamma Ray, 106990K (6 July 2018); doi: $10.1117 / 12.2312877$

SPIE Event: SPIE Astronomical Telescopes + Instrumentation, 2018, Austin, Texas, United States 


\title{
The fourth flight of CHESS: spectral resolution enhancements for high-resolution FUV spectroscopy
}

\author{
Nicholas Kruczek $^{\mathrm{a}}$, Nicholas Nell ${ }^{\mathrm{a}}$, Kevin France ${ }^{\mathrm{a}, \mathrm{b}}$, Keri Hoadley ${ }^{\mathrm{c}}$, Brian Fleming ${ }^{\mathrm{a}}$, Stefan \\ Ulrich $^{\mathrm{a}}$, Alexander D. Miller ${ }^{\mathrm{d}}$, Arika Egan ${ }^{\mathrm{a}}$, Emily Witt ${ }^{\mathrm{a}}$, and Robert Kane ${ }^{\mathrm{e}}$ \\ ${ }^{a}$ Laboratory for Atmospheric and Space Physics, University of Colorado, UCB 600, Space \\ Science Building (SPSC), 3665 Discovery Drive, Boulder, CO, USA 80309 \\ ${ }^{\mathrm{b}}$ Center for Astrophysics and Space Astronomy, University of Colorado, 389 UCB, Boulder, \\ CO, USA 80309 \\ ${ }^{\mathrm{c}}$ Cahill Center for Astrophysics, California Institute of Technology, 1200 East California \\ Boulevard, Mail Code 278-17, Pasadena, CA 91125, USA \\ ${ }^{\mathrm{d}}$ Arizona State University School of Earth and Space Exploration, 781 South Terrace Road, \\ Tempe, AZ, USA 85281 \\ eSEAKR Engineering, 6221 S. Racine Circle, Centennial, CO, 8011
}

\begin{abstract}
In this proceeding, we describe the scientific motivation and technical development of the Colorado Highresolution Echelle Stellar Spectrograph (CHESS), focusing on the hardware advancements and testing of components for the fourth and final launch of the payload (CHESS-4). CHESS is a far ultraviolet rocket-borne instrument designed to study the atomic-to-molecular transitions within translucent cloud regions in the interstellar medium. CHESS is an objective echelle spectrograph, which uses a mechanically-ruled echelle and a powered (f/12.4) cross-dispersing grating; it is designed to achieve a resolving power $\mathrm{R}>100,000$ over the band pass $\lambda \lambda$ 1000-1600 Å. CHESS-4 utilizes a 40 mm-diameter cross-strip anode readout microchannel plate detector, fabricated by Sensor Sciences LLC, to achieve high spatial resolution with high global count rate capabilities $(\sim$ $\mathrm{MHz}$ ). An error in the fabrication of the cross disperser limited the achievable resolution on previous launches of the payload to $\mathrm{R} \sim 4000$. To remedy this for CHESS-4, we physically stress the echelle grating, introducing a shallow toroidal curvature to the surface of the optic. Preliminary laboratory measurements of the resulting spectrum show a factor of $4-5$ improvement to the resolving power. Results from final efficiency and reflectivity measurements for the optical components of CHESS-4 are presented, along with the pre-flight laboratory spectra and calibration results. CHESS-4 launched on 17 April 2018 aboard NASA/University of Colorado Boulder sounding rocket mission 36.333 UG. We present flight results for the observation of the $\gamma$ Ara sightline.
\end{abstract}

Keywords: echelle spectrograph, far-ultraviolet, rocket-borne, astrophysics

\section{INTRODUCTION}

The Colorado High-Resolution Echelle Stellar Spectrograph (CHESS) ${ }^{1-6}$ is a rocket-borne astronomical instrument that first launched from White Sands Missile Range (WSMR) aboard NASA/CU mission 36.285 UG on 24 May 2014 (CHESS-1). The second flight of the experiment was aboard the NASA/CU mission 36.297 UG on 21 February 2016 (CHESS-2) and the third took place on 26 June 2017 aboard NASA/CU mission 36.323 UG. This paper presents information on the instrument optics, alignment, calibration, and flight results of CHESS-4, which was flown aboard the NASA/CU mission 36.333 UG on 17 April 2018 from the Reagan Test Site on Roi-Namur in the Republic of the Marshall Islands. This section covers the scientific objectives and instrumental design of the CHESS experiment.

Further author information: (Send correspondence to Nick Kruczek)

E-mail: Nicholas.Kruczek@colorado.edu, Telephone: +1 3037358214

Space Telescopes and Instrumentation 2018: Ultraviolet to Gamma Ray, edited by Jan-Willem A. den Herder, Shouleh Nikzad, Kazuhiro Nakazawa, Proc. of SPIE Vol. 10699, 106990K · (c) 2018 SPIE CCC code: $0277-786 X / 18 / \$ 18 \cdot$ doi: $10.1117 / 12.2312877$

Proc. of SPIE Vol. 10699 106990K-1 


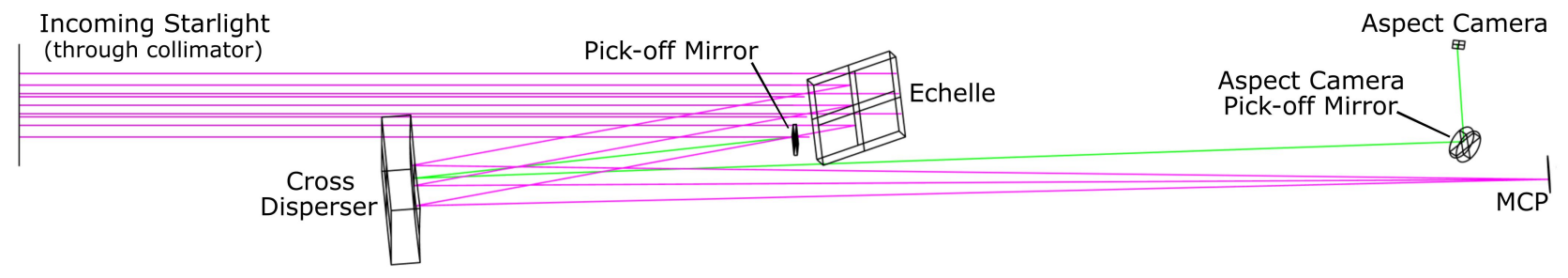

Figure 1. The Zemax ray trace of CHESS, including the secondary aspect camera system. The mechanical collimator reduces stray light in the line of sight and feeds starlight to the echelle. The echelle disperses UV light into highdispersion orders, which are focused by the cross disperser onto the detector plane. The green lines trace light with $\lambda=$ $5500 \AA$ through to the aspect camera. The pink lines trace light with $\lambda=1216 \AA$ to the microchannel plate detector.

\subsection{Scientific Objectives}

Translucent clouds reside in the transition between the diffuse (traditionally defined as $\left.\mathrm{A}_{V}<1\right)$ and dense $\left(\mathrm{A}_{V}>\right.$ 3) phases of the interstellar medium (ISM). It is in this regime where the ultraviolet (UV) portion of the average interstellar radiation field plays a critical role in the photochemistry of the gas and dust clouds that pervade the Milky Way galaxy and provide the raw materials for future star and planet formation. A powerful technique for probing the chemical structure of translucent clouds is to combine measurements of $\mathrm{H}_{2}$ with knowledge of the full carbon inventory (C I, C II, and CO) along a given line of sight. It has been argued that an analysis of the carbon budget should be the defining criterion for translucent clouds, rather than simple measurements of visual extinction. ${ }^{7}$ Moderate resolution 1000-1120 ^ spectra from FUSE and higher resolution data from HST-STIS have been used to show that many of these sightlines have $\mathrm{CO} / \mathrm{H}_{2}>10^{-6}$ and $\mathrm{CO} / \mathrm{C} \mathrm{I} \sim 1$, consistent with the existence of translucent material in the framework of current models of photodissociation regions in the ISM. . $^{8,9}$

The CHESS experiment is designed to study translucent clouds with its combination of bandpass and spectral resolution. The far ultraviolet (FUV; 1000-1600 $\AA$ ) bandpass contains absorption lines of $\mathrm{H}_{2}(1000-1120 \AA)$, C II (1036 and $1335 \AA$; however we note that saturation effects can complicate the interpretation of these lines), C I (several between 1103-1130, 1261, $1561 \AA$ ), and the A-X, B-X, C-X, and E-X bands of CO $(<1550 \AA)$. High resolution $(\mathrm{R}>100,000)$ is required to resolve the velocity structure of the $\mathrm{C}$ I lines and the rotational structure of $\mathrm{CO}$. High-resolution is therefore essential to the accurate determination of the column density of these species. ${ }^{10}$ The FUV also provides access to many absorption lines of metals, such as iron, magnesium, silicon, and nickel, allowing for an exploration of the depletion patterns in translucent clouds. CHESS, with its high-resolution and large bandpass, including wavelengths shorter than $1150 \AA$, is well-suited to study translucent clouds and will help create an observational base for models of the chemistry and physical conditions in interstellar clouds.

The fourth and final flight of CHESS continued to build upon our program on the local interstellar medium (begun on the NASA/CU mission 36.271 UG/SLICE ${ }^{11-13}$ and continued on the three previous flights of CHESS $^{1,6,14}$ ) with a detailed study of the interstellar material and on-going interactions in the line of sight of $\gamma$ Arae $(\gamma$ Ara). $\gamma$ Ara is a B1I star at $d=689 \mathrm{pc}$ and was chosen because it is known to display a variable and equatorially enhanced stellar wind ${ }^{15}$ that could potentially host a population of ro-vibrationally excited $\mathrm{H}_{2}$ at the wind/ISM interface. CHESS is ideally suited to study this molecular population, providing insights into a key catalyst in the chemistry of the diffuse and translucent ISM and the thermal and turbulent environments of both atoms and molecules in our Galactic neighborhood.

\subsection{Instrument Design}

CHESS is an objective $\mathrm{f} / 12.4$ echelle spectrograph. The instrument design ${ }^{4}$ included the development of two novel grating technologies and flight-testing of a cross-strip anode microchannel plate (MCP) detector. The highresolution instrument was designed to achieve resolving powers $\geq 100,000 \lambda / \Delta \lambda$ across a bandpass of 1000-1600 $\AA .{ }^{5}$ The operating principle of the instrument is as follows: 
- A mechanical collimator, consisting of an array of $10.74 \mathrm{~mm} \times 10.74 \mathrm{~mm} \times 1000 \mathrm{~mm}$ anodized aluminum tubes, provides CHESS with a total collecting area of $40 \mathrm{~cm}^{2}$, a field of view (FOV) of $1.37^{\circ}$, and allows on-axis stellar light through to the spectrograph.

- A square echelle grating (ruled area: $102 \mathrm{~mm} \times 102 \mathrm{~mm}$ ), with a designed groove density of 87 grooves $/ \mathrm{mm}$ and angle of incidence (AOI) of $63^{\circ}$, intercepts and disperses the FUV stellar light into higher diffraction terms $(\mathrm{m}=200-127)$. To improve the spectral resolution of the instrument, we mechanically shaped the surface of this grating to provide additional focusing power. This is discussed in more detail in $\S 2.1$ and $\S 3.2$.

- Instead of using an off-axis parabolic cross disperser, ${ }^{16}$ CHESS employs a holographically-ruled cross dispersing grating with a toroidal surface figure and ion-etched grooves, maximizing first-order efficiency. ${ }^{5}$ The cross disperser is ruled over a square area $(100 \mathrm{~mm} \times 100 \mathrm{~mm})$ with a groove density of 351 grooves $/ \mathrm{mm}$ and was designed to have a surface radius of curvature $(\mathrm{RC})=2500.25 \mathrm{~mm}$ and a rotation curvature $(\rho)$ $=2467.96 \mathrm{~mm}$. The grating spectrally disperses the echelle orders and corrects for grating aberrations. ${ }^{17}$

- The cross-strip MCP detector ${ }^{18,19}$ is circular in format, $40 \mathrm{~mm}$ in diameter, and capable of total global count rates of $\sim 10^{6}$ counts/second. The cross-strip anode allows for high resolution imaging, with the location of a photoelectron cloud determined by the centroid of current readout from nine anode "fingers" along the $\mathrm{x}$ and $\mathrm{y}$ axes. Figure 2 displays an example of a laboratory spectrum taken with the fully integrated and aligned CHESS instrument.

The CHESS experiment includes a second optical path that directs light to an aspect camera that is used to align the spectrograph to the target during calibrations and flight. The aspect camera system uses two pick-off mirrors and the cross disperser to direct zeroth-order light to an intensified Xybion camera. We also flew a side pointing Nocturn camera to assess its flight performance as a potential aspect camera. This low-light camera is an off-the-shelf device manufactured by Photonis and could be useful as a replacement for the Xybion aspect camera currently commissioned aboard sounding rocket missions.

\subsection{Mechanical Spectrograph Structure}

The opto-mechanical structure of CHESS-4 is nearly identical to that presented in Ref. 1. Below, we outline information and specifications about the structure and optical mount designs for CHESS. A SolidWorks rendering of the spectrograph and electronics sections of CHESS is provided in Figure 3.

CHESS is an aft-looking payload housed in three 17.26" diameter rocket skins. It is split into two sections: a vacuum (spectrograph) section and an electronics section. The two sections are separated by a hermetic bulkhead. The exterior of the payload went through a slight redesign for CHESS-4 to account for the planned water landing of the instrument. The electronics skin was lengthened from 50.80 to $62.87 \mathrm{~cm}$ and its two doors were fitted with watertight covers. The Nocturn pointed out of one of the doors using the same modified port flown on 36.323. An additional hermetic bulkhead was installed at the end of the electronics section to ensure it remained watertight. The overall length of the payload is $292.10 \mathrm{~cm}$ from mating surface to mating surface and the weight of the payload is $361 \mathrm{lbs}$.

The vacuum section uses two $113.36 \mathrm{~cm}$ long rocket skins with hermetic joints. The only mechanical mechanism on CHESS (other than the NASA Sounding Rockets Operations Contact (NSROC)-supplied shutter door) is a manual butterfly valve attached along the $180^{\circ}$ line on the aft skin. This allows for the evacuation of the experiment throughout development, integration, and pre-flight activities, in order to safeguard the sensitive optical coatings and detector photocathode. A carbon-fiber optical metering structure is attached to the aft side of the hermetic bulkhead and suspends the aspect camera, mechanical collimator, echelle grating, and cross-disperser in place. The space frame is comprised of three aluminum disks attached to five $2.54 \mathrm{~cm}$ diameter $\mathrm{x} 182.88 \mathrm{~cm}$ long carbon fiber tubes. The detector is mounted with a hermetic seal to the forward side of the $1.00 "$ thick hermetic bulkhead and faces aft, into the vacuum section.

The collimator is a set of $10.74 \mathrm{~mm} \times 10.74 \mathrm{~mm} \times 1000 \mathrm{~mm}$ long black-anodized aluminum square tubes bonded together. An aluminum mounting flange is bonded at the center of the assembly and secured to the aft-most disk of the space frame. 


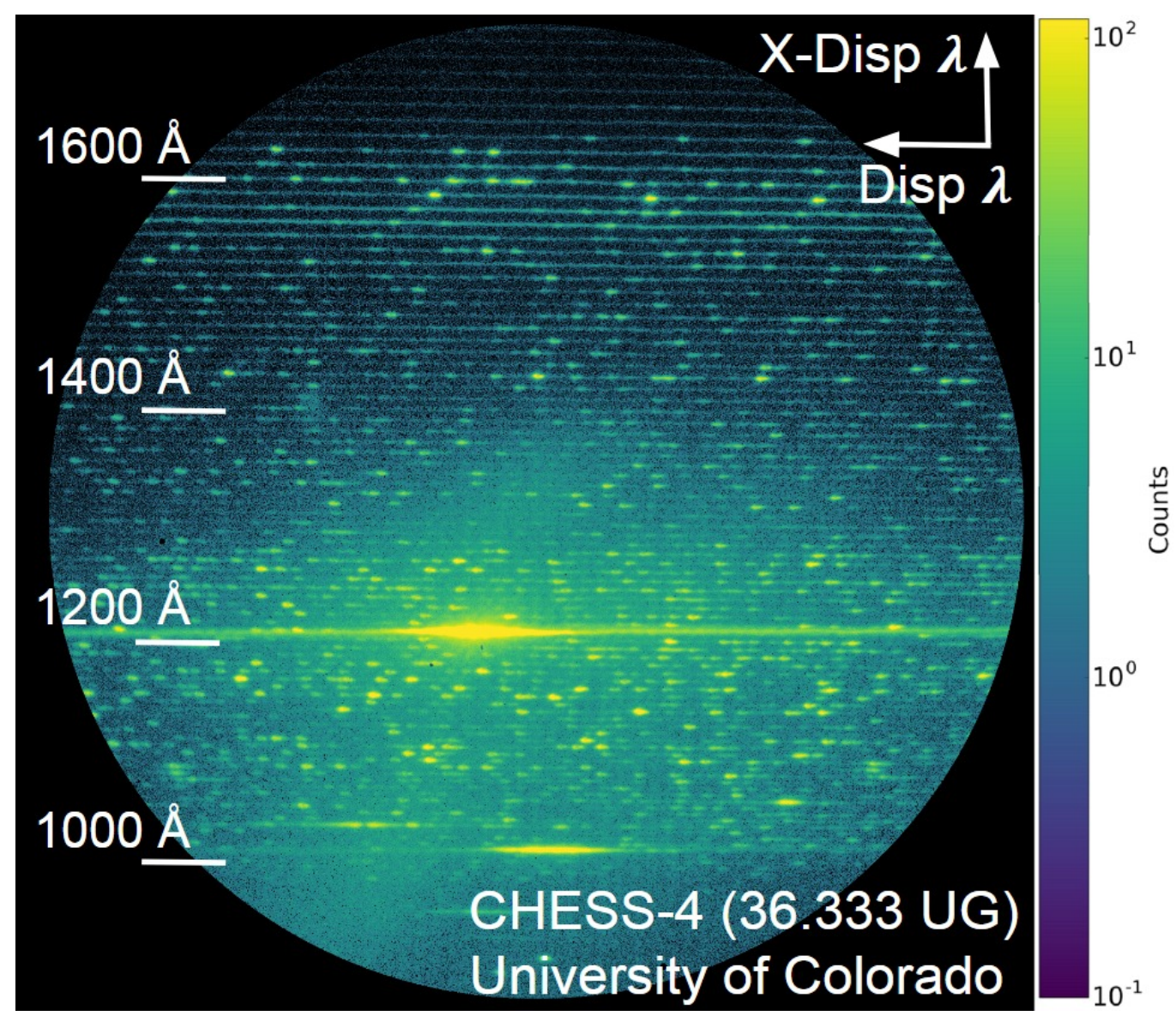

Figure 2. A false-color representation of the laboratory echellogram of CHESS-4 in the pre-flight configuration, binned to $4096 \times 4096$ pixels. The black/purple represents little to no counts in the binned pixel location, while yellow represents emission lines from atomic and molecular hydrogen. Overlaid are arrows showing the direction of dispersion from the echelle and cross disperser and the tick marks showing the approximate location of different spectral regions. The final laboratory calibration image contains $\sim 70$ million photon counts.

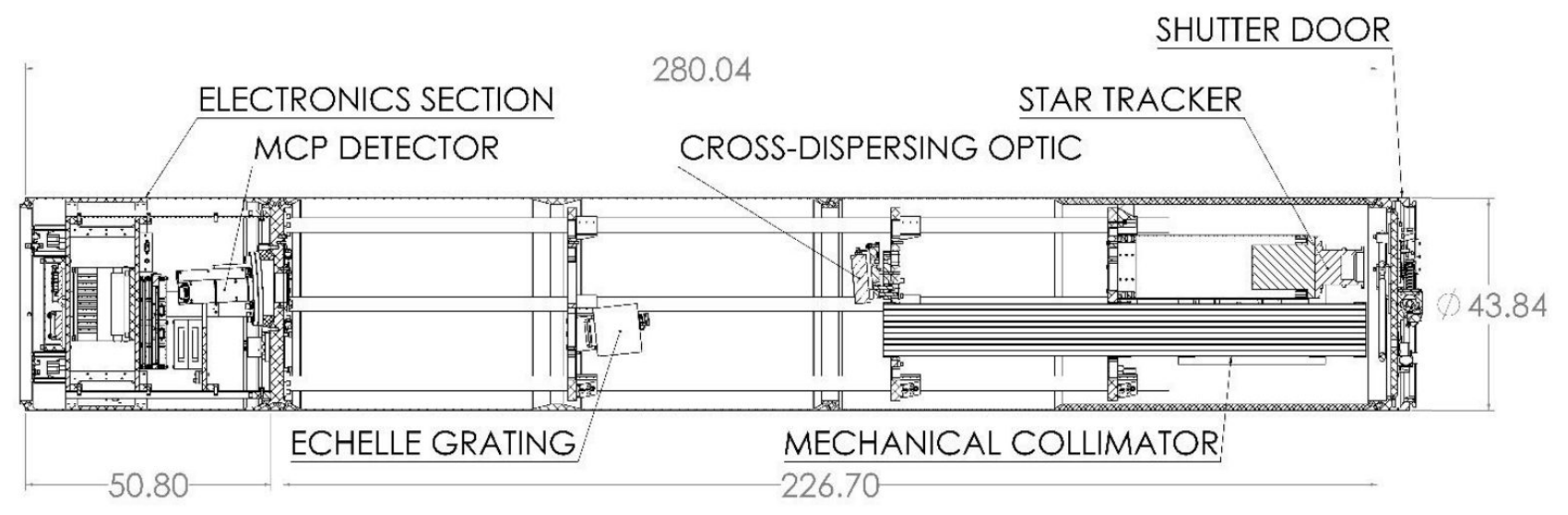

Figure 3. A SolidWorks rendering of the spectrograph and electronics sections of CHESS. Labeled are relevant spectrograph structures and optical components referenced in $\S 1.3$. All stated quantities are in units of centimeters. 


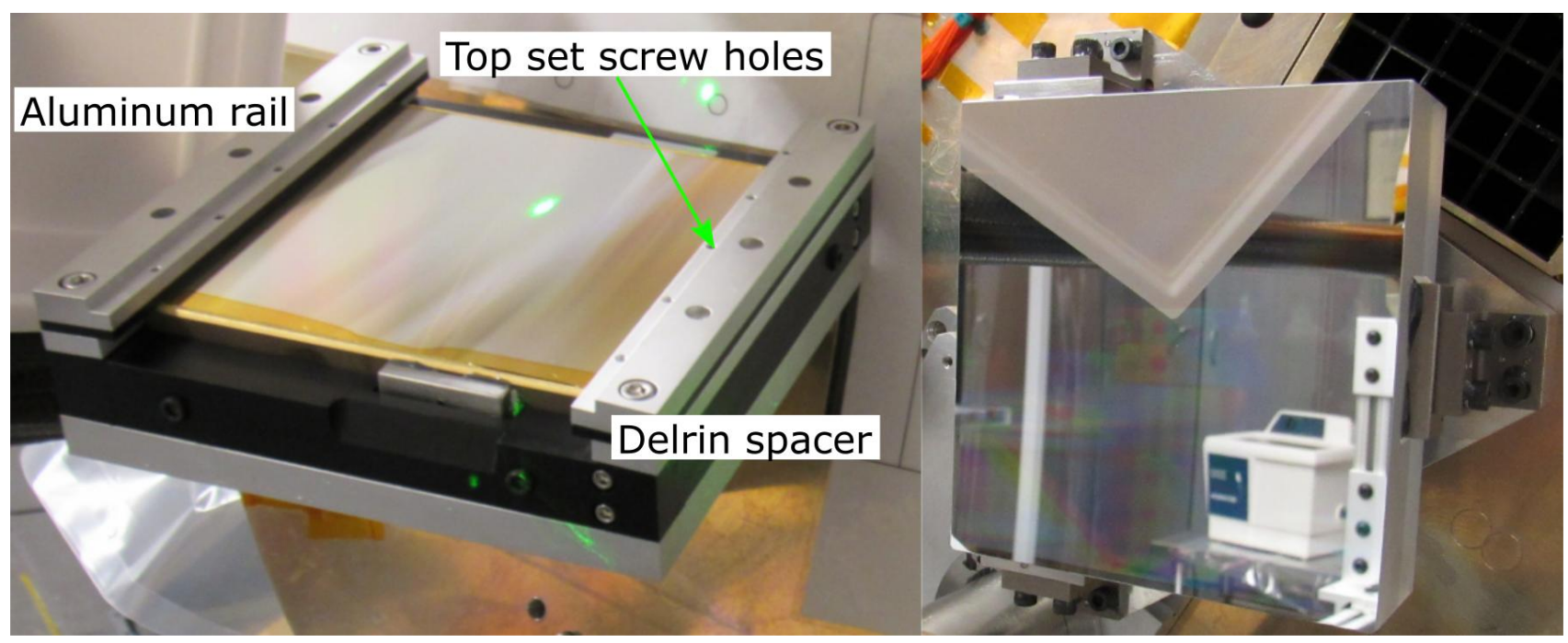

Figure 4. The CHESS echelle (left) and cross disperser (right).

The echelle grating in CHESS-4 is a rectangular block made of Zerodur (110 mm x $110 \mathrm{~mm}$ x $16 \mathrm{~mm}$ thick). The grating is housed in an open-faced aluminum and Delrin box that secures the optic and provides the mounting points for set screws that are used to shape the echelle. This redesigned mount is discussed in more detail in §2.1. The box is secured to an aluminum mount, which is set to the desired AOI and is attached to the forward most disk of the space frame.

The cross disperser is a block of fused silica (100 mm x $100 \mathrm{~mm}$ x $30 \mathrm{~mm}$ thick) with a toroidal surface. Three Invar pads are bonded to the neutral plane of the optic, nearly $120^{\circ}$ apart, and are connected to three titanium flexures. The flexures are attached to an aluminum mounting plate and affixed to the middle disk of the space frame. The flexures prevent the surface of the optic from warping due to stress transfer from a coefficient of thermal expansion mismatch in mounting components.

\section{OPTICAL PERFORMANCE}

\subsection{Echelle Grating}

The CHESS echelle was designed to be a $100 \mathrm{~mm}$ x $100 \mathrm{~mm}$ x $0.7 \mathrm{~mm}$ silicon wafer with a groove density (g) of 69 grooves $/ \mathrm{mm}$ and AOI $(\alpha)=67^{\circ}$. As detailed in Refs. 1 and 14, attempts to fabricate an echelle that met these specifications using lithographic and e-beam etching lead to gratings with peak efficiencies of $5 \%$, four times smaller than the minimum acceptable value for CHESS-1. We instead opted for mechanically-ruled gratings in subsequent launches. These gratings can have higher efficiencies but historically display higher inter-order scatter. ${ }^{20}$

The CHESS-4 echelle was fabricated by Richardson Gratings (formerly Milton-Roy; Rochester, NY) and had a designed $\mathrm{g}=87$ grooves $/ \mathrm{mm}$ and $\alpha=63^{\circ}$ ruled over a $102 \mathrm{~mm} \mathrm{x} 102 \mathrm{~mm}$ area. It was the same grating that was flown on CHESS-3. During initial alignments (see $\S 3$ ), we found that these values were closer to $\mathrm{g}=89.5$ grooves $/ \mathrm{mm}$ and $\alpha=63.22^{\circ}$ which were still within the CHESS tolerances. The echelle is coated with a layer of aluminum $(\mathrm{Al})$ to maximize reflectivity. The aluminum is coated with a layer of lithium fluoride (LiF) to prevent oxidation.

During the integration of CHESS-2, we noticed that the cross disperser needed to move closer to the MCP than expected to reach the focus of the orders. Post-flight testing revealed that the optic had been ruled $90^{\circ}$ off. This means that the RC and the $\rho$ were interchanged, making it impossible to simultaneously focus the spectral lines and orders and limiting the spectral resolution of the instrument. To help improve the instrument resolution for CHESS-4, we shaped the echelle grating by precisely torquing set screws at various points on the optic. This process required a new grating mount that could facilitate the different set screw positions. 


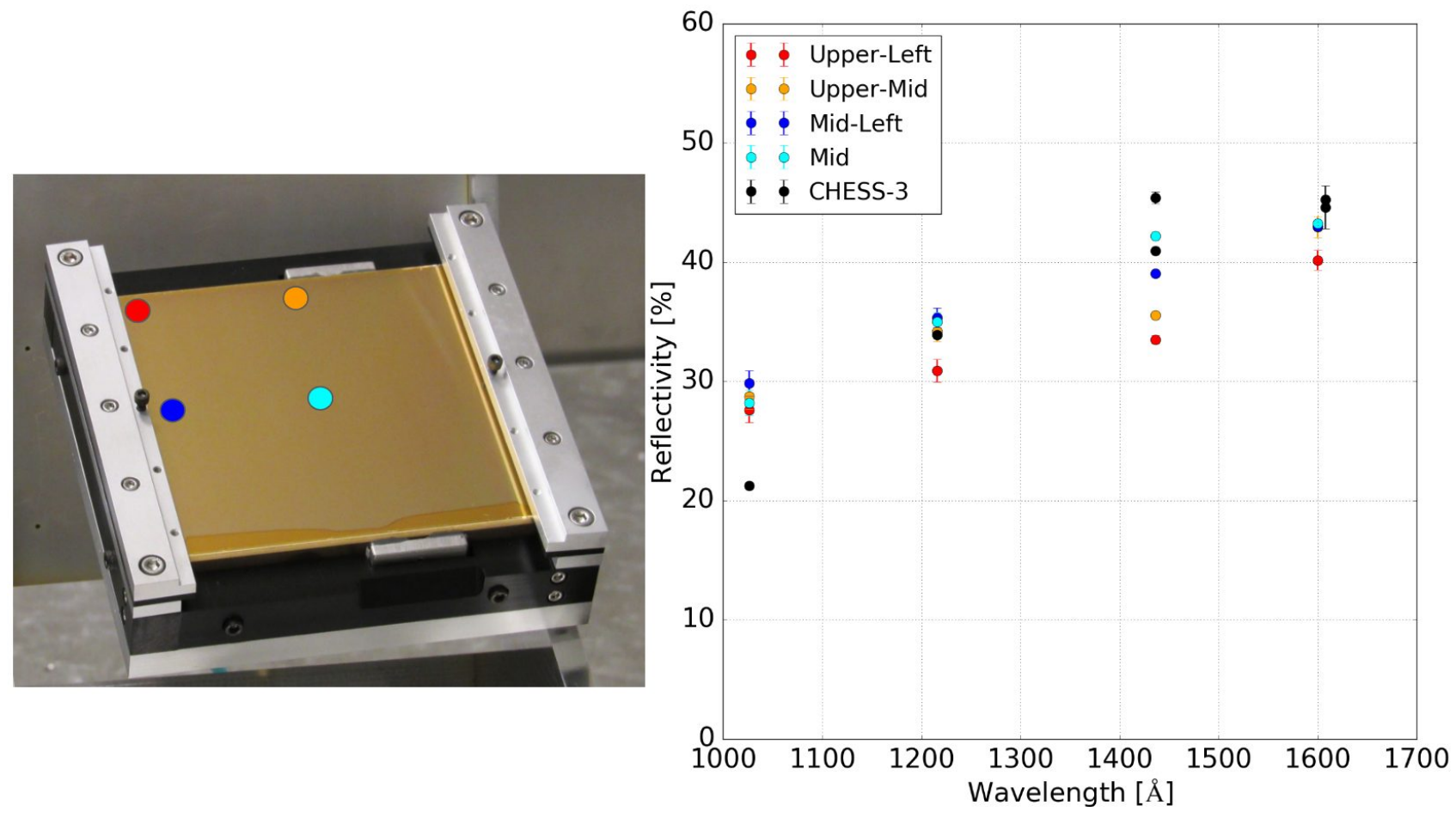

Figure 5. Left: The echelle in the reflectivity chamber. The four colored spots indicate the different positions where measurements were taken. The orange and blue spots are located at the highest and lowest points on the optic. For reference, one of the bottom sets screws was roughly located under the orange circle, the other was in the same spot on the opposite edge of the grating. Right: The measured reflectivities at the four points shown on the left, as well as the reflectivites of the unbent echelle from CHESS-3, for reference.

This grating mount was based on the secondary flight housing used on the FORTIS sounding rocket ${ }^{21}$ and can be seen in Figures 4 and 5. It is comprised of an aluminum base and Delrin box. The grating is held in the box by aluminum rails, with Delrin spacers underneath, that run along the sides of the optic. The grating sits in the box on top of a square Delrin spacer. To shape the optic, each aluminum rail has five holes for set screws that can be torqued into the Delrin beneath (see Figure 4). The Delrin acts as a buffer layer that prevents direct contact between the metal screw and Zerodur optic and helps distribute the pressure applied by the screw. There are two additional holes that pass up through the bottom aluminum layer into the lower Delrin spacer that are used to apply pressure to the bottom of the optic, facilitating the creation of a concave bend (see Figure 5).

To ensure that the shaped grating surface did not impact its efficiency, we induced a roughly "flight-like" curvature in it, using two set screws on top and two on the bottom, and then measured its reflectivity at four different spots that spanned the range of surface heights. The resulting reflectivites are plotted in Figure 5 . We did not see any significant impact to the overall efficiency as a function of position on the grating. The one exception is that there was a larger variation in the reflectivities at $1446 \AA$. Due to the consistency of the other points and the lower signal produced at this wavelength, we suspect that is more indicative of uncertainties in our setup, such as light source variability and detector alignment, than an actual difference in the grating efficiency.

\subsection{Cross Disperser Grating}

The CHESS cross disperser grating is a $100 \mathrm{~mm} \times 100 \mathrm{~mm} \times 30 \mathrm{~mm}$ fused silica optic with a toroidal surface profile. The toroidal surface shape separates the foci of the spatial and sagittal axes of the dispersed light. The optic is designed to first focus light spatially onto the detector, then spectrally behind the detector, ensuring no foci at the locations of either the ion repeller or quantum efficiency (QE) grids. The cross dispersing optic is a novel type of imaging grating that represents a new family of holographic solutions and was fabricated by Horiba 
Jobin-Yvon (JY). The line densities are low (351 lines per mm, difficult to achieve with the ion-etching process), and the holographic solution allows for more degrees of freedom than were previously available with off-axis parabolic cross dispersing optics. The holographic ruling corrects for aberrations that otherwise could not be corrected via mechanical ruling. The grating is developed under the formalism of toroidal variable line spacing gratings ${ }^{17}$ and corresponds to a holographic grating produced with an aberrated wavefront via deformable mirror technology. This results in a radial change in groove density and a traditional surface of concentric hyperboloids from holography, like those used in ISIS ${ }^{22}$ and $H S T / \mathrm{COS}^{23}$

Figure 6 shows the efficiency of the cross disperser for the $\mathrm{m}=-1$ order. The $\mathrm{Al}+\mathrm{LiF}$ coating was not reapplied between flights, instead the optic was stored in a nitrogen purge cabinet when not in use. We did not see any significant degradation in the performance of the optic between the two flights. The cross disperser is effective at dispersing most of the on-axis light into the $\mathrm{m}= \pm 1$ orders and suppressing the $\mathrm{m}=0$ order because of the characteristic sinusoidal groove profiles created via the ion-etching procedure at JY. Additionally, at optical wavelengths, the reflectivity of the $\mathrm{m}=0$ order becomes comparable to the $\mathrm{m}= \pm 1$ orders. This allowed us to build the secondary camera system to track the movements of our optical axis and target acquisition during flight.

\subsection{Cross-Strip Anode Microchannel Plate Detector}

The cross-strip MCP detector was built and optimized to meet the CHESS spectral resolution specifications at Sensor Sciences. ${ }^{18,19}$ The detector has a circular format and a diameter of $40 \mathrm{~mm}$. The detector uses two plates made from lead silicate glass, each containing an array of $10 \mu \mathrm{m}$ diameter channels. The plates are stacked on top of one another and are arranged in a "chevron" configuration. The top plate is coated with an opaque cesium iodide (CsI) photocathode, which provides detective quantum efficiency (DQE) $=15-35 \%$ at FUV wavelengths. When UV photons strike the photocathode to release photoelectrons, the photoelectrons are accelerated down the channels by an applied high voltage $(\sim 3100 \mathrm{~V})$. Along the way, they collide with the walls of the channels, which produces a large gain over the initial single photoelectron. The DQE across the CHESS bandpass is plotted in Figure 6 against the efficiency measurements of the flight echelle and cross disperser for CHESS-4. To help maintain the throughput of the instrument, the CsI photocathode was reapplied by Sensor Sciences for the final launch.

The 2015 NASA Cosmic Origins Program Annual Technology Report emphasized that the technology readiness level (TRL) for large format, high count rate, and high QE MCP detectors needs to improve for future UV space missions. One of the goals of the CHESS instrument is to demonstrate the flight performance of the cross strip anode design to raise the TRL level to 6, which was achieved on 36.285 and 36.297. The cross-strip anode MCP detector was re-flown and performed reliably once more on 36.333, handling count rates of $\sim 125,000$ photons/second for the entire science exposure of CHESS-4.

We also collaborated with the NASA Jet Propulsion Laboratory (JPL) and Arizona State University (ASU) to flight qualify a delta-doped high purity p-channel CCD that could exhibit broadband response from NUV to NIR for future NASA missions. JPL fabricated the detector by processing existing fully depleted high purity thick p-channel CCD wafers that were procured from Lawrence Berkeley National Laboratory under a separate effort. JPL applied their end-to-end post-fabrication back illumination processing including delta-doping to produce the detector. No further FUV optimization using antireflection coatings for this CCD was incorporated. The device was tested at JPL, using a qualified and calibrated quantum efficiency setup equipped with a vacuum monochromator and a National Institute of Standards and Technology (NIST)-calibrated photodiode ${ }^{24}$ exhibiting the expected typical delta-doped CCD QE in the CHESS spectral range. ${ }^{25,26}$ The calibrated device was delivered to ASU, where a focal plane assembly was built and optimized for the chip. The entire focal plane assembly is housed in a steel tube with a conflat flange designed to be mounted to the rear bulkhead of CHESS. The CCD chip, focal plane assembly, and controller, as received from ASU in May 2017, are shown in Figure 7. We measured the DQE of the focal plane assembly using our in-house detector calibration chamber, using a vacuum monochromator and a NIST-calibrated photodiode. ${ }^{27}$ The focal plane assembly payload displayed lower than anticipated DQE across CHESS bandpass, which was traced to hydrocarbon contamination in the focal plane array vacuum housing. The payload performance was insufficient to meet the flight science goals of CHESS- 4 . 

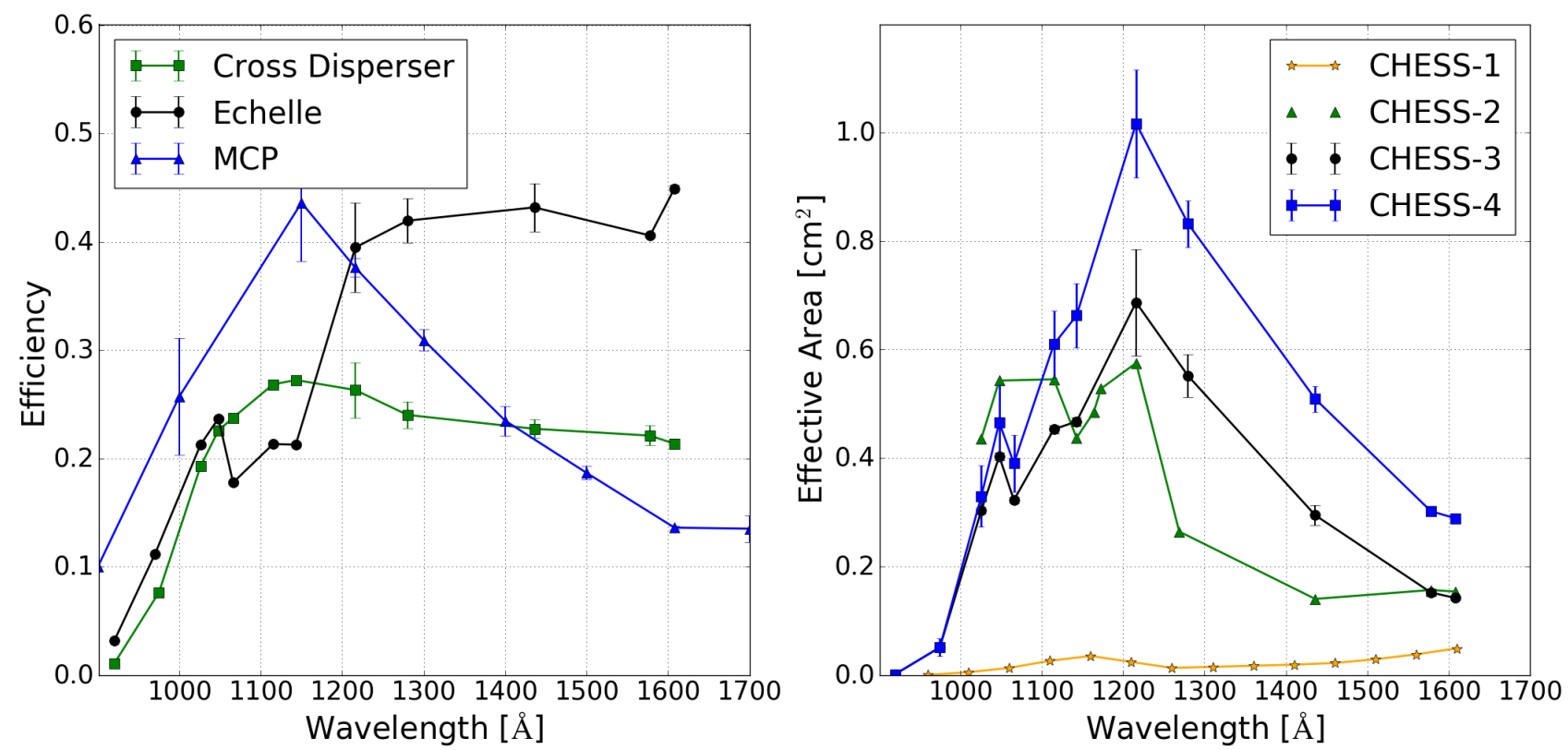

Figure 6. Left: Performance (for each grating: peak order reflectivity, and for the detector: DQE) of all optical components of CHESS-4. Our in-house measurements of the MCP DQE end at 1100 A. We used values provided by Sensor Sciences Inc. to extend the curve down to $900 \AA$. Right: A summary of the effective areas for all four CHESS launches. The gains in CHESS-4 spectrum were achieved by reapplying the CsI photocathode to the MCP.

\section{INSTRUMENT ALIGNMENTS AND CALIBRATIONS}

\subsection{Alignment and Focusing}

The alignment process for CHESS has been described in great detail by Ref. 1; please refer to this document for specifics and pictures of the process. Instead of reiterating the entire procedure in detail, we list the steps taken to align CHESS-4 and focus the echellogram for flight:

- The CHESS gratings were designed to have grating parameters that allow for optical wavelength solutions. Grating solutions were modeled in Zemax for green $(532 \mathrm{~nm})$ and violet $(405 \mathrm{~nm})$ wavelengths. Print-outs of the expected spot positions for each color were used to roughly align the echelle to the cross disperser and then align the cross disperser to the detector.

- For FUV calibrations, the instrument is aligned to an external vacuum system to feed the spectrograph with UV light. We used the aspect camera system to align the instrument to the vacuum chamber. A small, square mirror was attached to a tip-tilt mount on one side of the echelle grating to intercept light. This mirror was directed to the center of the cross disperser. Zeroth-order optical light off the cross disperser intercepted another small, flat mirror at the detector bulkhead, which steered the light to the aspect camera. During alignments, white light was tracked with the aspect camera until the proper alignment position was found.

- Linear vacuum actuators were installed behind the cross dispersing optic to control the tip, tilt, and piston motions of the grating. Using these stages, we centered the echellogram on the detector and focused the spectrum using small actuator steps while the entire payload was under vacuum.

The above alignments were first performed with the flat echelle installed in payload, to ensure that all other instrument components were aligned and operating as anticipated. Figure 8 shows the focus curves for the spectral lines and order widths in this initial configuration. The final position of the cross disperser was determined by the location of the narrowest order width, not spectral width. While a narrow spectral width 


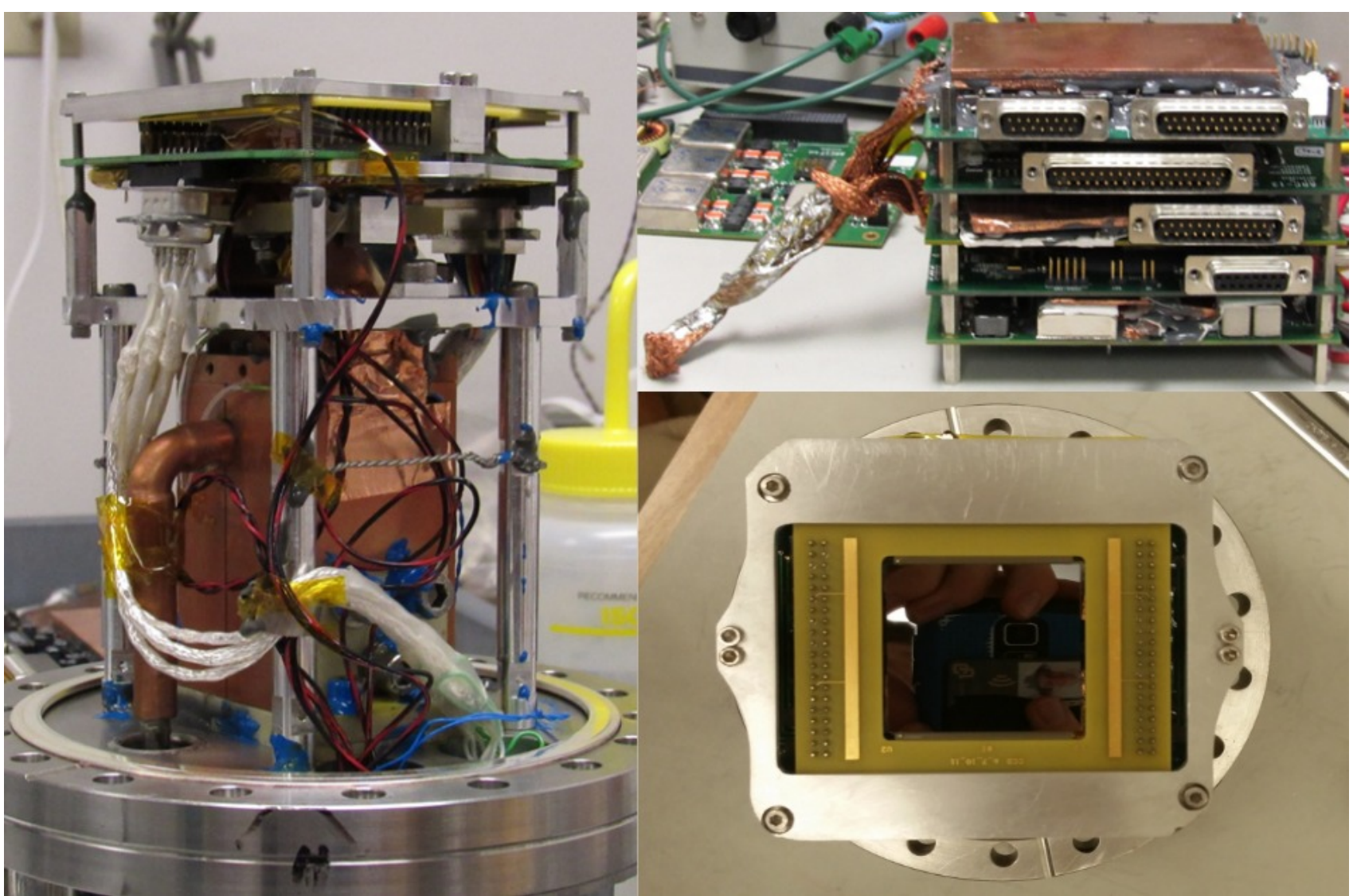

Figure 7. The ASU CCD payload components. The CCD focal plane assembly (left), the CCD controller (top right), and the JPL delivered delta doped CCD (bottom right).

would maximize the resolution of the instrument, the resulting overlap between neighboring orders would make the extraction of the one-dimensional (1D) spectrum difficult, if not impossible. This position was used as a reference point when focusing the instrument once the shaped echelle was installed.

\subsection{Spectral Resolution Enhancement}

After the initial alignment of the payload using the flat echelle, we uninstalled the mount and the optic and began the process of inducing the new curvature. To determine what shape was needed, we assumed a toroidal curvature and ran a damped least squares optimization within Zemax that minimized the overall spot radius at three different wavelengths: 1026,1147 , and $1400 \AA$. These wavelengths were chosen to favor the blue-end of the spectrum more heavily, since it contains the $\mathrm{H}_{2}$ absorption features. Figure 9 shows a comparison of the line spread functions (LSFs) between the unbent echelle and the Zemax optimized echelle bend, which was found to have $\mathrm{R}=2.55 \times 10^{5} \mathrm{~mm}$ and $\rho=-3.49 \times 10^{5} \mathrm{~mm}$.

A Zygo GPI XP Fizeau Interferometer was used to measure the surface curvature for various combinations of screws and torque specifications. Torques were applied using a torque wrench that allowed for steps as small as $1 \mathrm{in.} \mathrm{oz.} \mathrm{Running} \mathrm{these} \mathrm{tests} \mathrm{on} \mathrm{the} \mathrm{flight} \mathrm{optic} \mathrm{was} \mathrm{not} \mathrm{possible} \mathrm{due} \mathrm{to} \mathrm{the} \mathrm{sensitivity} \mathrm{of} \mathrm{its} \mathrm{coating} \mathrm{as} \mathrm{well} \mathrm{as}$ the complications inherent to measuring a ruled surface interferometrically. Instead, we used an unruled replica of the echelle that was purchased from Richardson Gratings. With the unruled replica, we were able to measure the curvature across the entirety of the surface of the optic, allowing us to determine what torque specifications were needed to best recreate the results predicted by Zemax. We tested several configurations of set screws including using only set screws on the bottom, only using screws on the top, as well as applying a gradient of torques across the top set screws to match the desired curvature along the edge. 

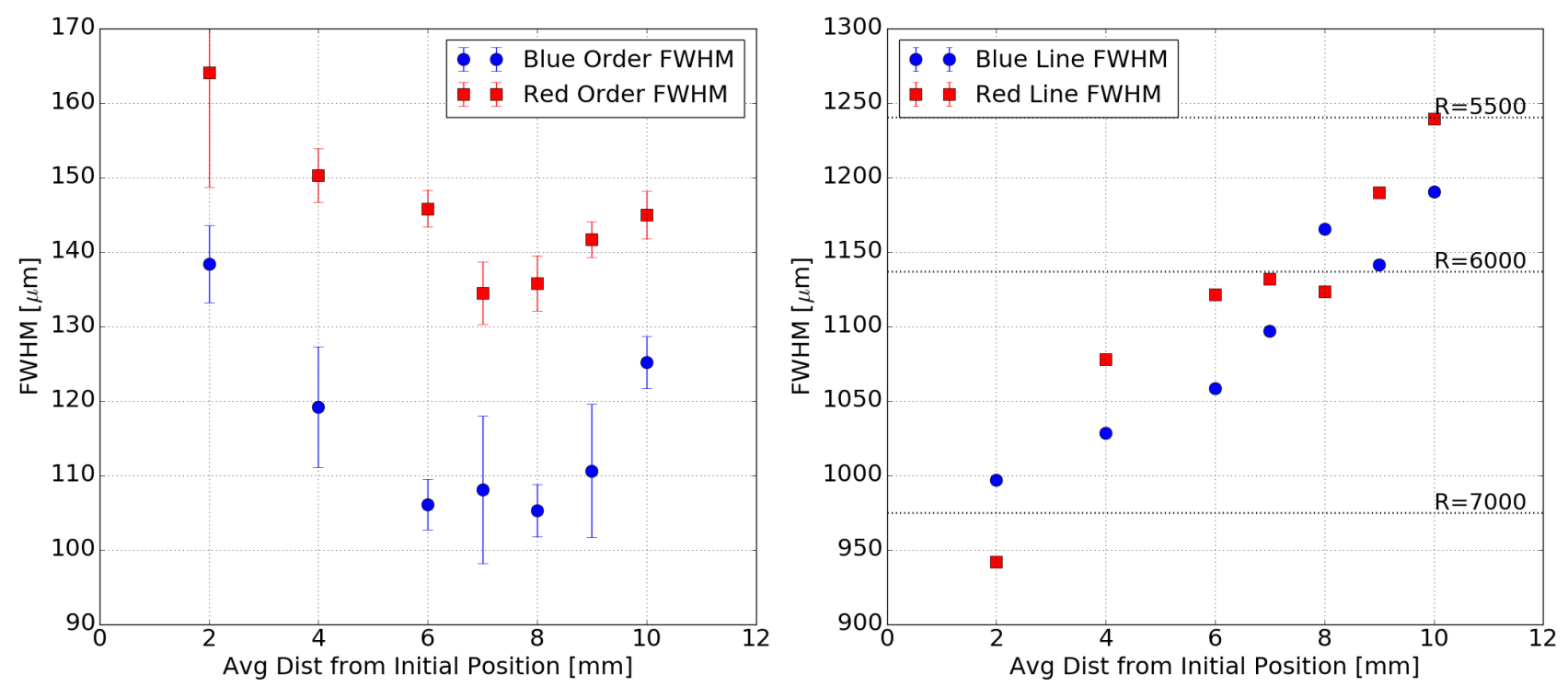

Figure 8. Left: The full width at half maximum (FWHM) of spectral orders as a function of distance from the initial position of the cross disperser when using the unbent echelle. A larger average distance from the initial position means the cross disperser was closer to the MCP detector. These curves were used to determine when the cross disperser reached its focal point. The blue circles show the width of an order on the blue end of the spectrum, red squares show the width of an order on the red end. Right: FWHMs of spectral features found in the orders used in the left plot. Estimated resolutions are shown as horizontal dotted lines.
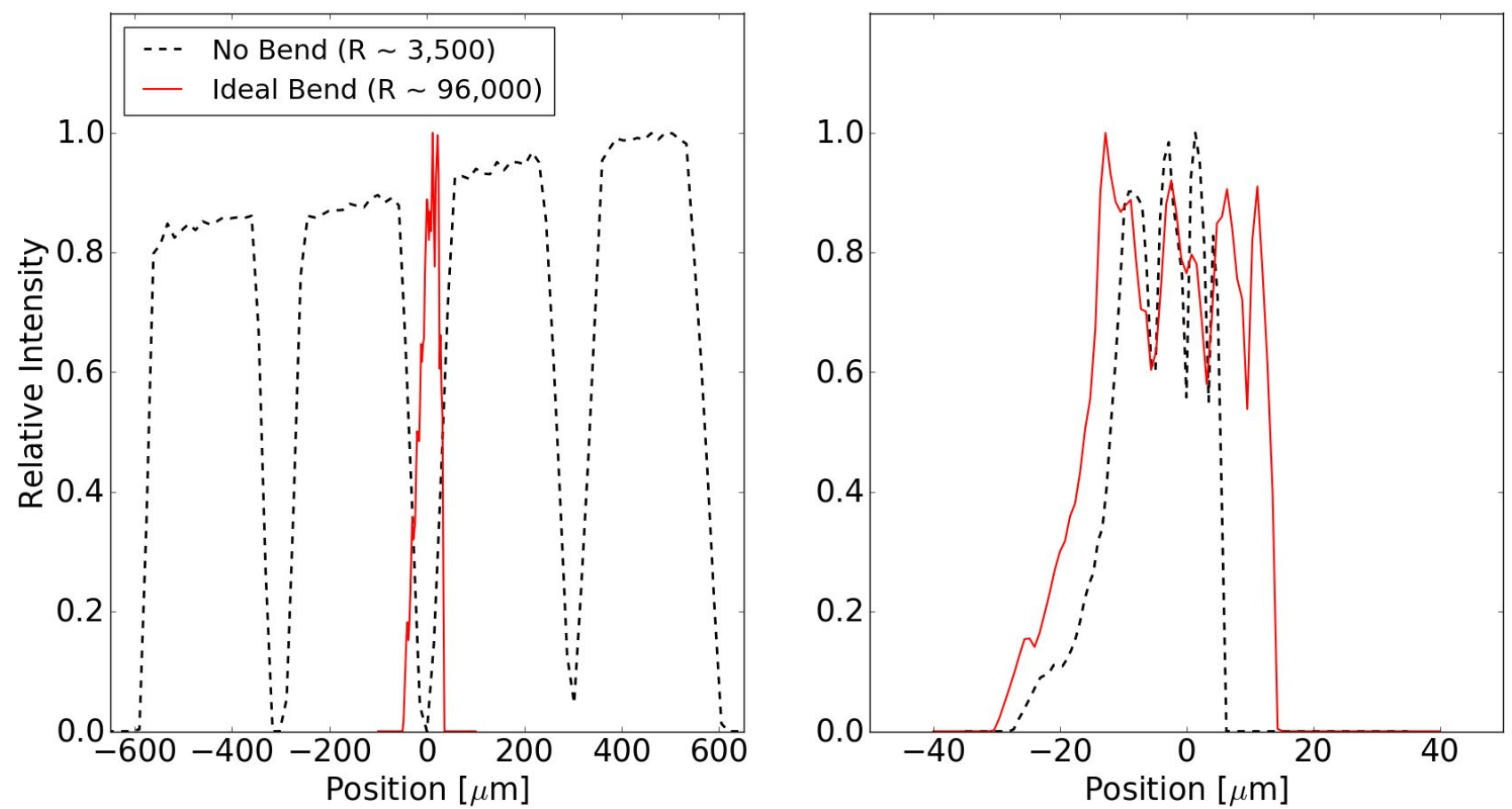

Figure 9. Left: The spectral line spread functions predicted by Zemax of the CHESS instrument for the flat echelle (black dashed line) and the Zemax optimized curved echelle (red line). Right: Same as left, but instead showing the order line spread functions. 

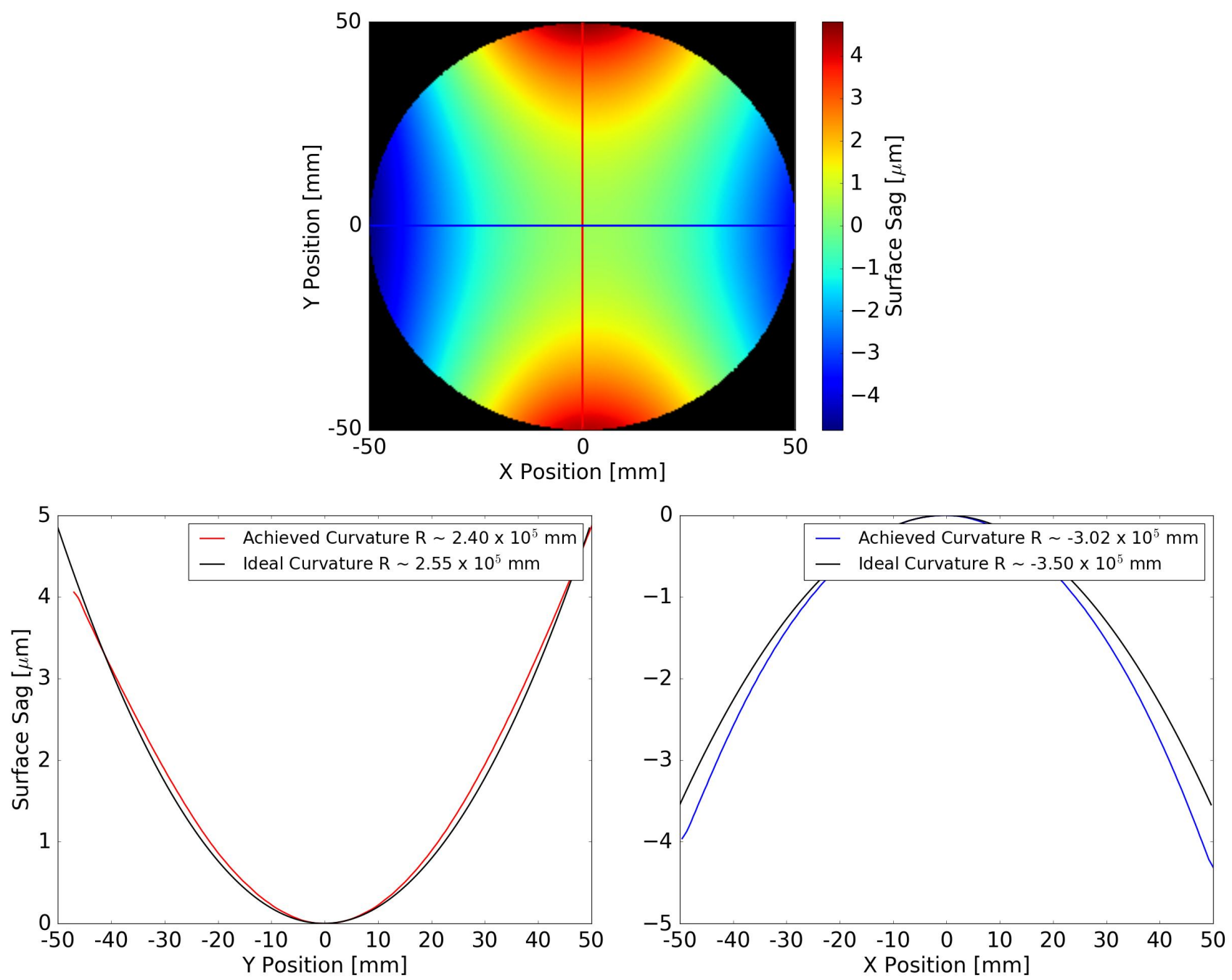

Figure 10. Top: The 2D surface profile of the unruled replica in the configuration that was the closest to the Zemax optimized result. The red and blue lines trace the paths of the 1D profiles shown in the lower plots. Left: The 1D profile running along the red vertical line in the top figure. Right: Same as left, but for the blue horizontal line in the top figure.

The set screw configuration that achieved the best results had four screws on top, one at each corner of the grating, that were torqued to $25 \mathrm{in}$. oz. There were also set screws in the two bottom holes that were torqued to $48 \mathrm{in.} \mathrm{oz.} \mathrm{The} \mathrm{2D} \mathrm{surface} \mathrm{profile} \mathrm{of} \mathrm{this} \mathrm{configuration,} \mathrm{as} \mathrm{measured} \mathrm{by} \mathrm{the} \mathrm{interferometer,} \mathrm{is} \mathrm{displayed} \mathrm{in}$ Figure 10. Also shown are two 1D surface profiles, these show the curvature along the the two perpendicular red and blue lines plotted over the 2D profile. The assumption of circular curvatures across the measured optic is not perfect, as demonstrated by the widening of the red profile, but we quote approximate radii of curvature for each profile that can be used as a rough comparison to the Zemax results.

Using these newly determined torque specifications, we replaced the grating replica with the flight optic and reinstalled it in the payload for final optimization of the curvature. The same procedure that was described in $\S 3.1$ was used to refocus the instrument each time a new torque specification was tested. To gain a better understanding of effect the top and bottom set screws had on the instrument LSF, we stepped through different torques while keeping one set fixed. Figure 11 shows the focus curves for three different torques on the top set screws. We found that while increasing the amount of torque on the top screws drove the order and spectral focuses towards each other, it also created wider spectral features. This meant that having no torque applied to the top set screws gave the narrowest spectral features at the order focus. 

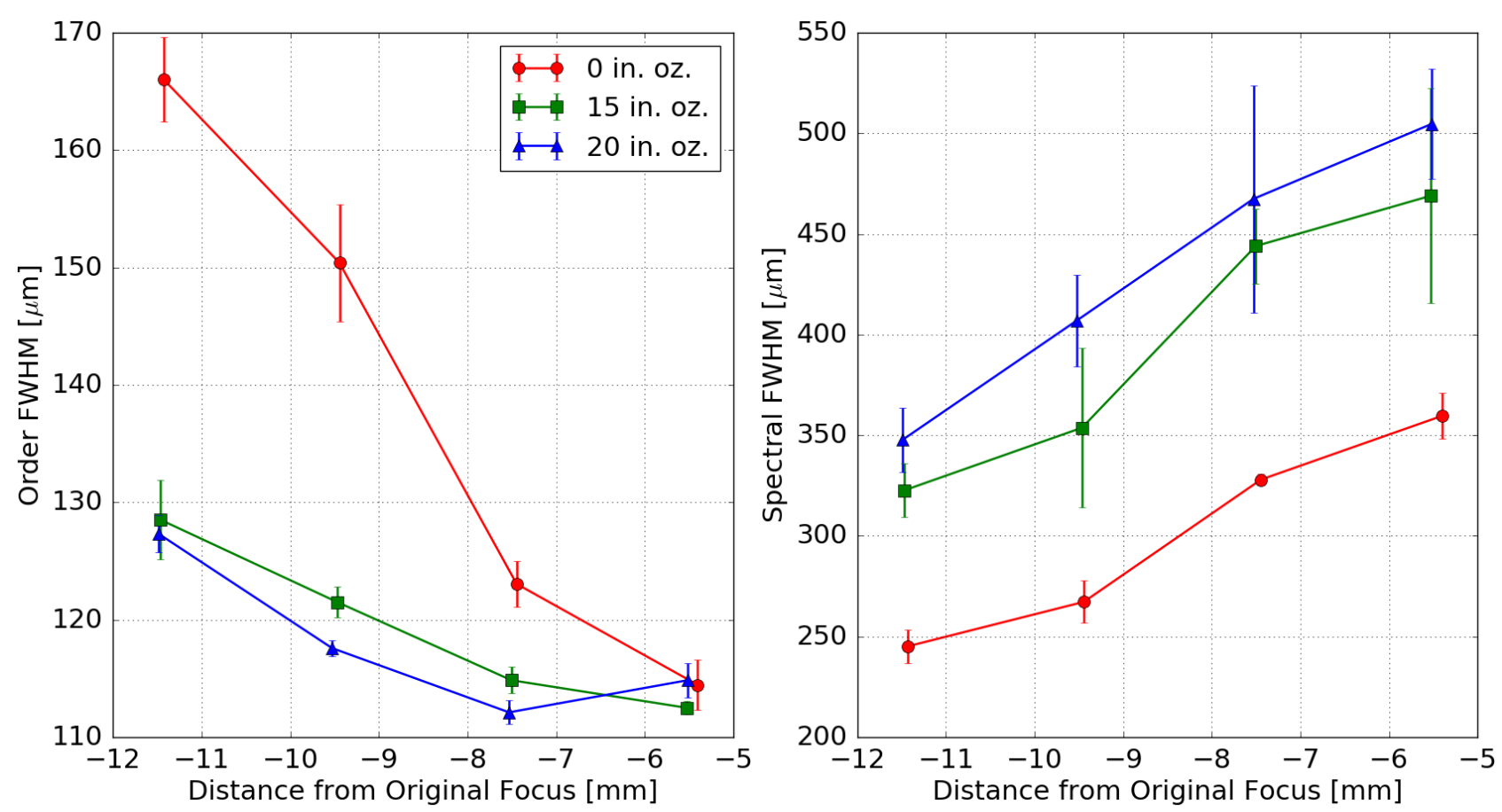

Figure 11. Focus curves of orders (left) and spectral features (right) as a function of cross disperser distance from the original focus of the instrument (i.e. when the flat echelle was installed). The three curves represent the FWHMs measured when different torques were applied to the four set screws on the top of the optic, with the bottom set screws held fixed at 48 in. oz.

Figure 12 shows the focus curves when different torque values were applied to the bottom set screws. We again found that increasing the torque value drove the two foci together, but this time the larger torque tended to create wider orders. When taking these measurements, the top set screws were installed but loosened before the first measurement. After performing the first measurement with 52 in. oz. of torque on the bottom screws, we found that the top set screws were tight due to the optic pushing up against them. After loosening them and retorquing the bottom screws, we repeated the measurement at $52 \mathrm{in.} \mathrm{oz.} \mathrm{While} \mathrm{this} \mathrm{configuration} \mathrm{resulted} \mathrm{in}$ generally wider orders, it did have the narrowest combined order and spectral feature width. Specifically, at $\sim-7$ $\mathrm{mm}$ we measured spectral features that were $\sim 150 \mu \mathrm{m}$ wide while maintaining orders that were $<130 \mu \mathrm{m}$. Since we expected further torquing the bottom set screws would make the orders too wide, we opted to stay with 52 in. oz. on the bottom set screws and no top set screws as our final flight configuration.

After final alignment and focus positions of the echelle and cross disperser were determined, long exposures using a hollow-cathode ("arc") lamp fed by $65 / 35 \%$ hydrogen/argon (H/Ar) mixture and air were taken for a complete sampling of $\mathrm{H}, \mathrm{H}_{2}, \mathrm{~N}$, and $\mathrm{O}$ emission lines in the CHESS bandpass. These spectra are used to characterize the $1 \mathrm{D}$ extracted spectrum, define the wavelength solutions of the instrument, and determine the LSFs across the bandpass.

Figure 13 shows a side-by-side comparison of the CHESS-3 and CHESS-4 pre-flight echellograms. The improvement in the spectral focus is immediately apparent. In addition to that, we repositioned the spectrum on the detector so that the peak echelle order was roughly centered. Both echellograms are co-additions of multiple exposures taken under vacuum. Each exposure was defined by how long we could run the full instrument configuration in vacuum without over-heating the electronics section, which typically lasted around 30 minutes. A large amount of scatter, that appears to be centered around Ly $\alpha$, is seen in the spectra. We expect that this is a symptom of the mechanically-ruled echelle, as discussed in $§ 2.1$. 

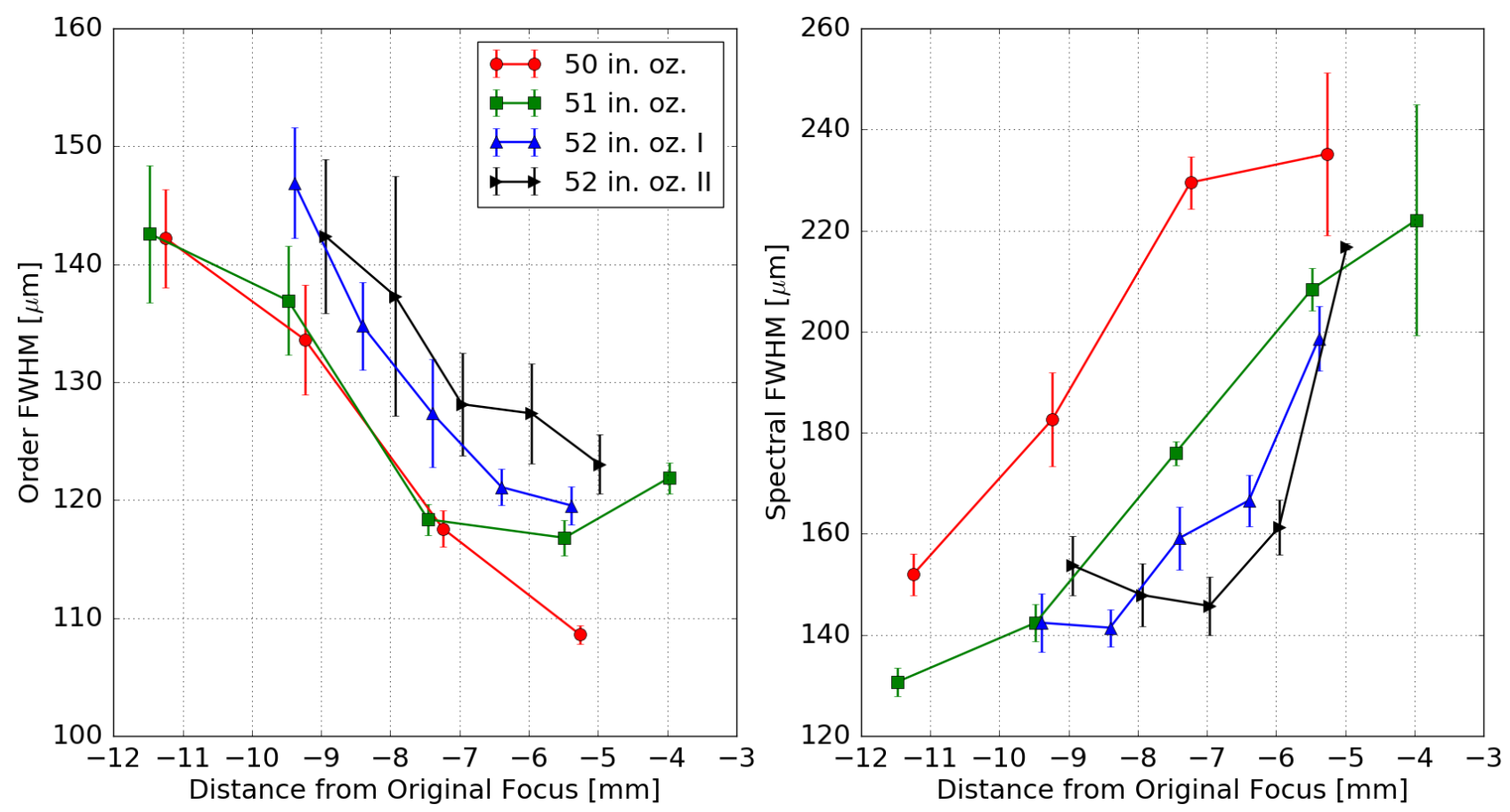

Figure 12. Focus curves of orders (left) and spectral features (right) as a function of cross disperser distance from the original focus of the instrument (i.e. when the flat echelle was installed). The four curves represent the FWHMs measured when different torques were applied to the two set screws on the bottom of the optic, with the top set screws loosened. Once reaching 52 in. oz. (blue triangles), we realized that the top set screws were still tight enough to have an impact on the curvature. After loosening them, we reran the focus curve at 52 in. oz. (black sideways triangles).

\subsection{Creating a 1D Spectrum}

Extracting the 1D spectra from the echellogram was accomplished through several steps. First, the echellogram had to be rotated very slightly $\left(\theta<1^{\circ}\right)$ to align the orders horizontally. The location of each order was then determined by summing (collapsing) all photon counts in the direction parallel to the orders, which created an "order spectrum" with peaks where orders were present and troughs at inter-order pixels. This exercise also determined the width of each order, which were $\sim 15$ pixels $(130 \mu \mathrm{m})$ wide in the CHESS-4 echellogram.

Once pixel locations and order widths were extracted, we collapsed a given order along the perpendicular axis, creating a 1D spectrum of each order. Across a majority of the spectrum, neighboring orders overlap in wavelength space. Common spectral features between neighbors simplified the stitching together of orders. To do this, we first accounted for the difference in $\Delta \lambda$ as a function order that is predicted by the grating equation by scaling each order to be $0.94 \times$ the length of its higher order neighbor. The 0.94 scale factor was determined through visual inspection of neighboring orders. We then identified two pairs of orders that contained strong overlapping emission features and used those to calculate a linear equation (see Equation 1) relating the amount of overlap to the average of the two order numbers $(m$ and $m+1)$.

$$
\text { overlap }=a \times \frac{m+(m+1)}{2}+b
$$

This equation was applied to the 88 identified orders to create a 1D spectrum in a scaled pixel space. Regions where orders overlap were summed together. The amount of overlap decreased towards the red-end of the spectrum, as a result of the increased dispersion predicted by the grating equation. This, combined with the fact that the MCP is circular in format and thus a smaller fraction of each order was captured on the detector at the red-end of the echellogram, means that we did not fully sample the red-end of the spectrum. The $\Delta \lambda$ scaling broke down at the longest wavelengths and we mitigated this effect by taking the $\log _{0.94}$ of the entire array. 


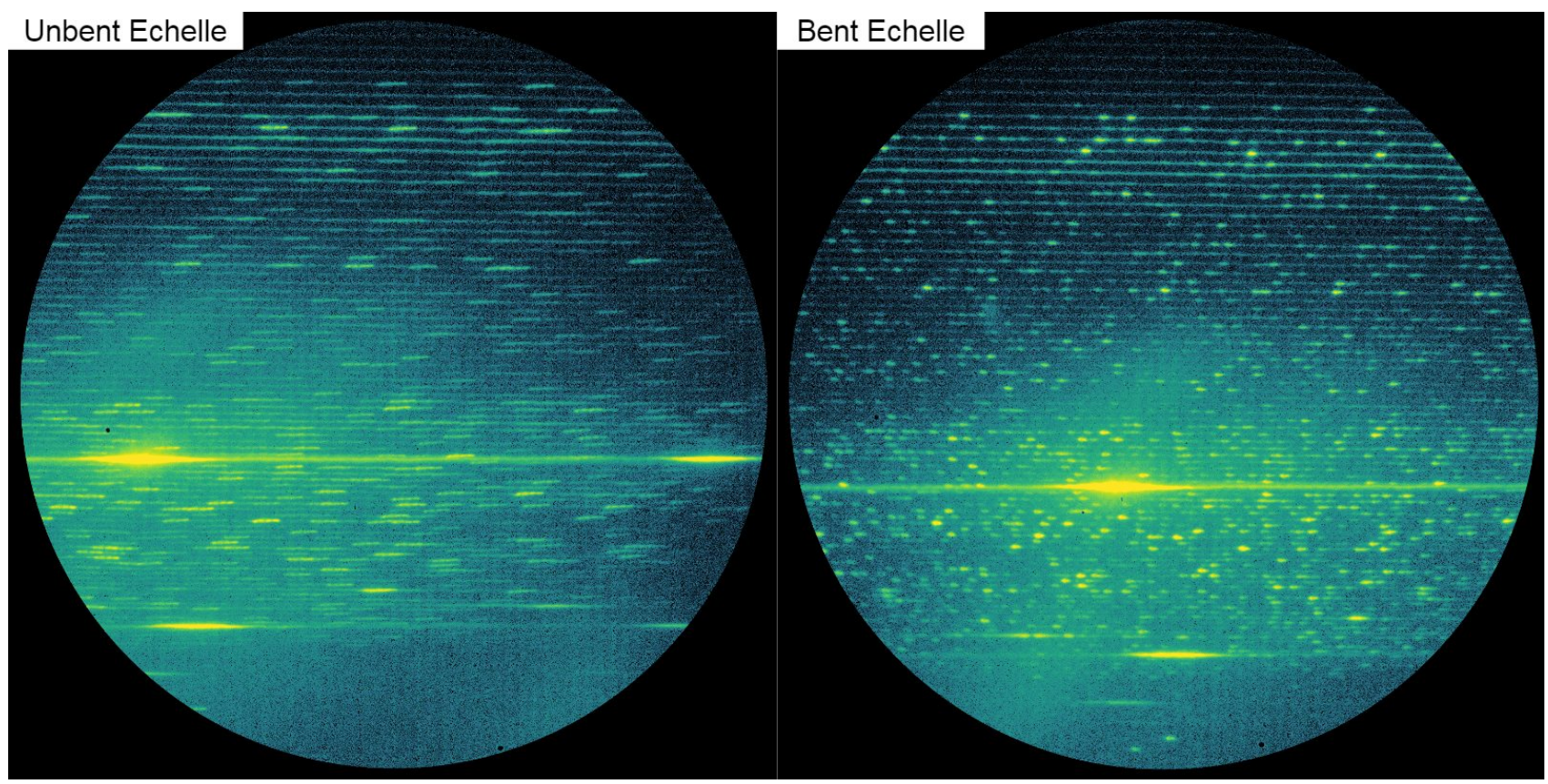

Figure 13. Raw images (trimmed for edge effects) of the CHESS-3 (May 2017, unbent echelle) and CHESS-4 (January 2018 , bent echelle) echellograms. The brightest feature in both images is HI-Ly $\alpha$ (1215.7 $\AA$ ). The other bright broad feature is HI-Ly $\beta$ (1025.7 $\AA$ ). HI-Ly $\gamma(972.5 \AA)$ is visible below the Ly $\beta$ feature. The large shifts in the spectrum were made so that the peak of the echelle order was better centered on the detector. The narrower spectral features in the CHESS-4 echelleogram are the result of the shaped echelle grating providing additional focusing power to the system.

We used the composition of air through the arc lamp to map out well-known atomic lines and their corresponding line centers in our 1D spectrum. These lines were common enough that we could sample most of the CHESS wavelength space from 1000-1500 A. To extend across the entire CHESS bandpass, we used the 1D spectrum generated using the $\mathrm{H} / \mathrm{Ar}$ arc lamp echellogram to identify $\mathrm{H}_{2}$ electron-impact lines at the red end of the spectrum $(\lambda>1510 \AA)$. In total, we mapped the locations of 18 air and $\mathrm{H}_{2}$ emission features between the air and $\mathrm{H} / \mathrm{Ar}$ spectra. Using the known wavelengths of each of the emission features, we fit a $6^{\text {th }}$-order polynomial to convert pixel position to wavelength across our 1D spectrum. The resulting wavelength solution for the pre-flight $\mathrm{H} / \mathrm{Ar}$ calibration spectrum is shown in Figure 14. Figure 15 displays a subsection of this spectrum along with a subsection of the pre-flight calibration spectrum from CHESS-3, demonstrating the improvement in the spectral LSFs that we achieved by introducing the shaped echelle.

\subsection{Spectral Resolution Determination}

After the wavelength solution was found for the pre-flight calibration echellogram, we determined the resolving power of the spectrum produced by CHESS-4. To do this, we created a multi-Gaussian fitting routine to capture each emission feature. While the features were much narrower for CHESS-4, they still typically required two Gaussians to adequately capture the entire shape. We defined the FWHM of an emission line by calculating the FWHM of each individual Gaussian and then measuring the distance between the center of the left hand peak minus its half width at half maximum (HWHM) to the center of the right hand peak plus $1 / 2$ of its HWHM. The resulting width is used to calculate the resolution of the spectral feature. Figure 16 shows the measured pre-flight resolution as a function of wavelength. We achieved an average resolution of $13,859 \pm 1,562$. This measurement was performed on data that was taken after the payload had been shipped to Wallops for preliminary integration. Prior to shipping, we did measure larger resolutions of $\mathrm{R} \sim 18,000$, but the shipping of the payload likely caused the shaped echelle to settle slightly. The $\mathrm{R} \sim 14,000$ better reflects the flight-like conditions and so we only provide that data here. 

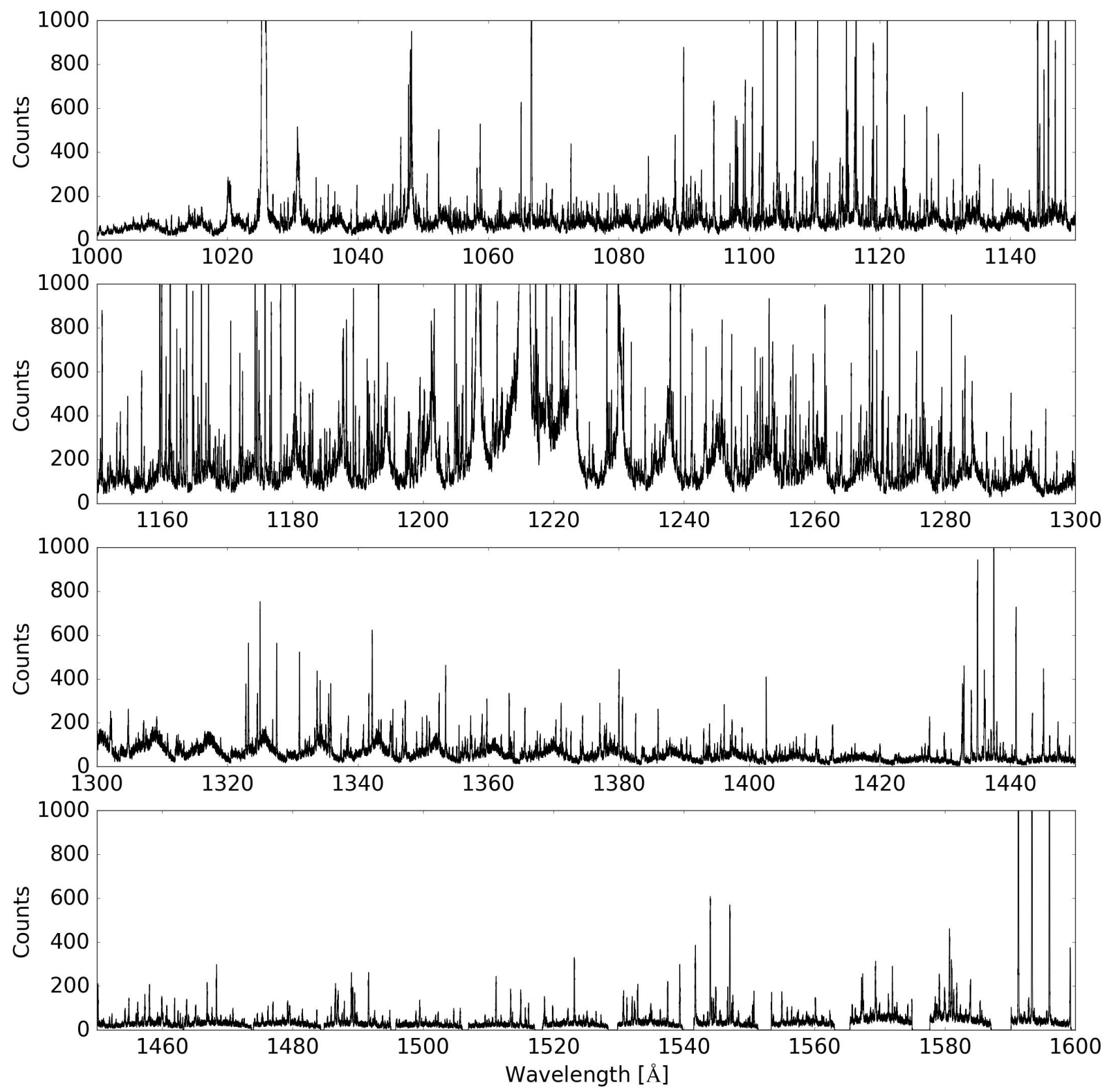

Figure 14. Complete first-order wavelength solution for the pre-flight CHESS-4 calibration spectrum, using H/Ar gas. As discussed in $\S 3.3$, neighboring orders no longer overlap starting around $1500 \AA$, resulting in gaps in the spectrum. 


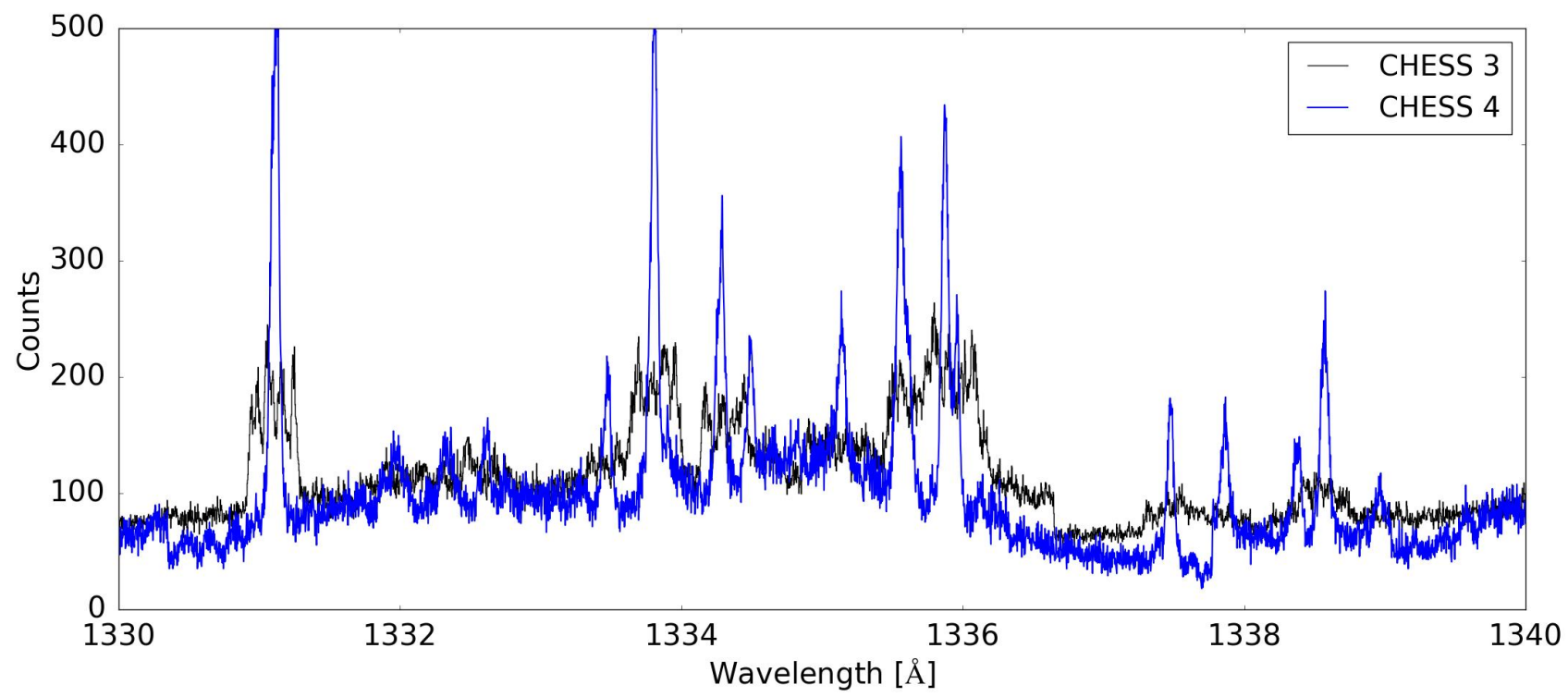

Figure 15. A comparison of the CHESS-3 pre-flight spectrum (black) and the CHESS-4 pre-flight spectrum (blue).

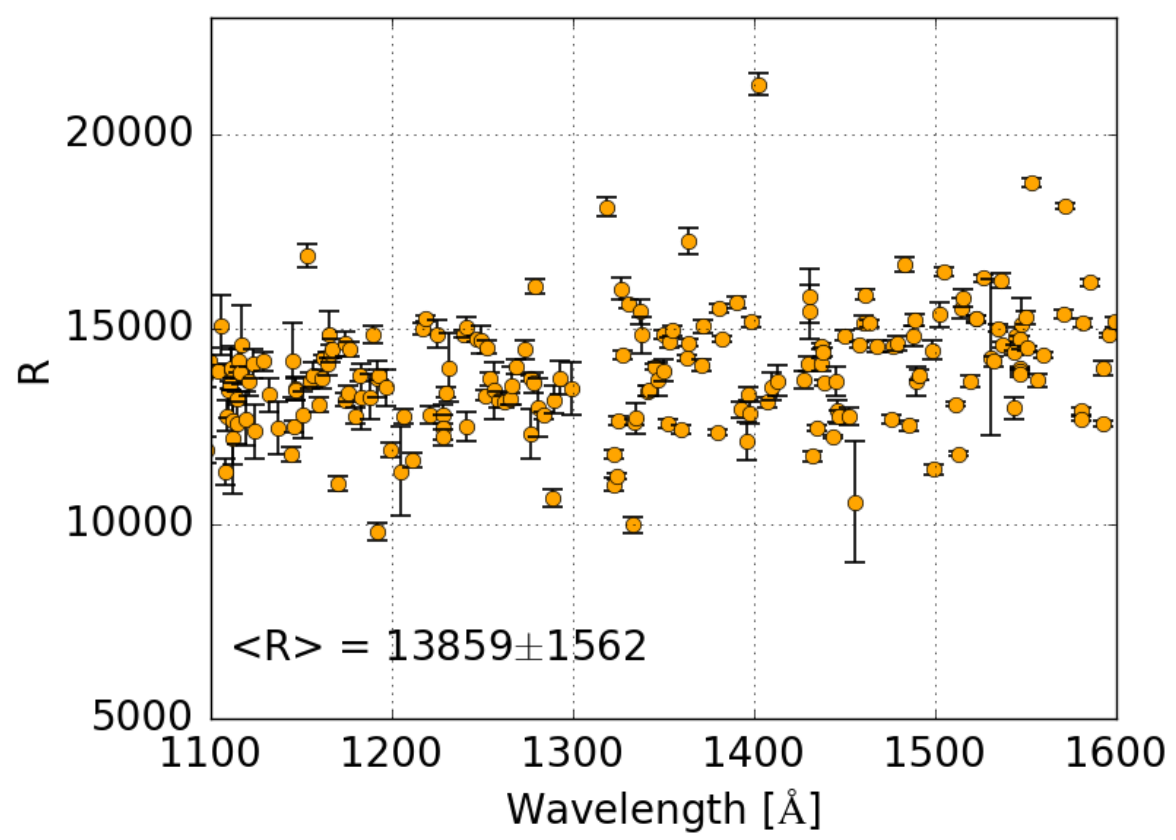

Figure 16. CHESS-4 pre-flight resolution. Each point comes from a line identified in the H/Ar calibration spectrum. The mean $(\langle R\rangle)$ and standard deviation of the distribution is included in the lower left. 


\section{CHESS-4 LAUNCH AND PRELIMINARY FLIGHT RESULTS}

CHESS-4 was brought to Wallops Flight Facility in late November 2017 for preliminary integration. The payload underwent various tests, including vibration, which required a means of determining alignment shifts before launch. To do this, we fit an electron lamp with a small collimating mirror and pinhole $(100 \mu \mathrm{m})$ to the shutter door. This lamp produced a spectrum based off of the gas that was flown into it and resulting spectral features were used to confirm optical alignment. After the integration, the payload was returned to CU for post-integration testing before being shipped to the launch site.

CHESS-4 was launched aboard NASA mission 36.333 UG from the Reagan Test Site on Roi-Namur in the Republic of the Marshall Islands on 17 April 2018 at 4:47 am MHT using a two-stage Terrier/Black Brant IX vehicle. The mission was deemed a comprehensive success. A single uplink maneuver was needed to initially align the star to the optical axis and we were able to integrate for $\sim 300$ seconds $(\mathrm{T}+105-420)$ on-target, with an approximate count rate of 125,000 photons/sec. After the 300 second exposure, we moved to an off-target calibration position where we took a $\sim 40$ second long exposure to obtain a measurement of the background Ly $\alpha$ and O I airglow for subtraction from the on-target spectrum.

Throughout the science exposure, we saw the star slowly drift across the aspect camera FOV, indicating that either our pointing was not stable or there was a component moving in our optical system. This motion was confirmed on our detector, where the location of the echelle orders moved as a function of time. To correct for this drift in the data, we binned the flight data as a function of time, using 5 -second wide bins. At each time step, we created an order spectrum (see §3.3) that was then cross-correlated with a reference spectrum that was chosen to be a 5-second wide bin from the middle of the exposure. Using the measured offset, the order spectrum could be shifted and then added into a new drift-corrected spectrum. This procedure was repeated for every bin and the final dark-subtracted, drift-corrected spectrum is shown in Figure 17.

The payload splashed down approximately 170 miles north of Roi-Namur and all recovery systems functioned as designed. Due to rough seas at the impact site, the recovery ship was unable to immediately remove the payload from the water, instead towing it $\sim 50$ miles to a calmer region where it could be brought aboard. Upon return to Roi-Namur, we found that the instrument held at sub-atmospheric pressures and the watertight skins successfully kept the CHESS instrument and electronics section dry. The payload was purged with nitrogen and stored for shipping back to Colorado.

\section{FUTURE WORK}

The CHESS-4 payload is currently still on Roi-Namur and is scheduled to return in late August 2018. Pending an initial inspection to ensure that all of the electronics are still functional and that there are no major sources of contamination in the payload, we plan to run through a suite of post-flight calibrations to determine if the payload alignment was maintained through launch and recovery and to measure the instrument efficiency after shipping. We will also attempt to measure the curvature that we achieved on the echelle, again using the interferometer.

Post-processing of the science data is on-going, with a focus on fitting the stellar continuum. Once the entire spectrum is normalized, it will be fit using a set of $\mathrm{H}_{2}$ optical depth templates ${ }^{28}$ to determine the column density and temperature of molecular hydrogen along the line of sight. A preliminary analysis over a short wavelength range has already been completed, and is shown in Figure 17. We will additionally be modeling a population of excited $\mathrm{H}_{2}\left(\mathrm{v}^{\prime \prime}=1\right)$ transitions to look for the predicted shocked-heated population of $\mathrm{H}_{2}(\S 1.1)$ within our spectrum.

\section{ACKNOWLEDGMENTS}

The authors would like to thank the students and staff at CU for their tremendous help in seeing CHESS-4 come to fruition. We would also like to thank the NSROC staff at WFF and on Roi-Namur for their tireless efforts that pushed us to a smooth launch. We thank all involved in the alternate CCD payload at JPL and ASU for their efforts. NN would like to thank Sugar Ray for their timeless melodies. This work was supported by NASA grants NNX13AF55G and NNX16AG28G to the University of Colorado. 

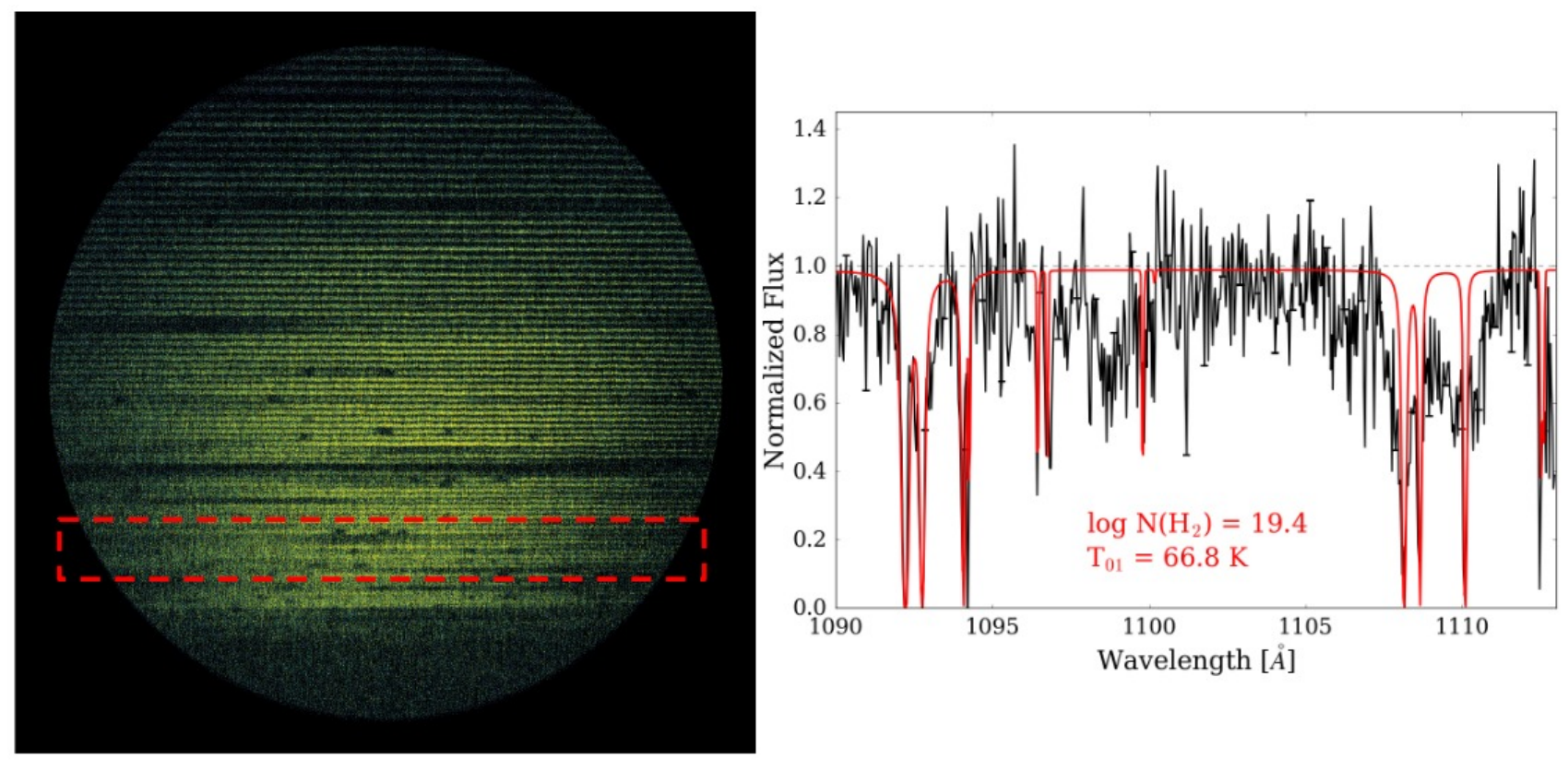

Figure 17. The flight data from 36.333 UG (CHESS-4). Left: The science echellogram of $\gamma$ Ara after an exposure time of $\sim 300$ seconds, with the background removed, the detector edge effects trimmed, and the drift correction applied. The echelle orders are stacked horizontally in the image, with order spectra easily distinguishable across most of the spectrum. This image has the same dispersion and cross-dispersion orientation as the calibration image (Figure 2). We are currently comparing this spectrum to the pre-flight calibrations to calculate any shifts in the spectrum during flight and extract a full 1D spectrum. The red box roughly shows the region where the data used in the right figure was extracted from. Right: A preliminary analysis of the flight data. The continuum normalized flight data is plotted in black. Overplotted in red is the ISM-like $\mathrm{H}_{2}$ absorption model that was fit to the data. The resulting $\mathrm{H}_{2}$ column density $\left(\log \mathrm{N}\left(\mathrm{H}_{2}\right)\right)$ and temperature $\left(\mathrm{T}_{01}\left(\mathrm{H}_{2}\right)\right)$ are also shown.

\section{REFERENCES}

[1] Hoadley, K., France, K., Nell, N., Kane, R., Schultz, T., Beasley, M., Green, J., Kulow, J., Kersgaard, E., and Fleming, B., "The assembly, calibration, and preliminary results from the Colorado high-resolution Echelle stellar spectrograph (CHESS)," in [Space Telescopes and Instrumentation 2014: Ultraviolet to Gamma Ray], Proc. SPIE 9144, 914406 (July 2014).

[2] France, K., Beasley, M., Kane, R., Nell, N., Burgh, E. B., and Green, J. C., "Development of the Colorado High-resolution Echelle Stellar Spectrograph (CHESS)," in [Space Telescopes and Instrumentation 2012: Ultraviolet to Gamma Ray], Proc. SPIE 8443, 844305 (Sept. 2012).

[3] Kane, R., Beasley, M., Green, J., Burgh, E., and France, K., "The opto-mechanical design of the Colorado High-resolution Echelle Stellar Spectrograph (CHESS)," in [Society of Photo-Optical Instrumentation Engineers (SPIE) Conference Series], Proc. SPIE 8145, 81450P (Sept. 2011).

[4] Beasley, M., Burgh, E., and France, K., "Colorado High-resolution Echelle Stellar Spectrograph (CHESS)," in [Space Telescopes and Instrumentation 2010: Ultraviolet to Gamma Ray], Proc. SPIE 7732, 773206 (July 2010).

[5] France, K., Hoadley, K., Fleming, B. T., Kane, R., Nell, N., Beasley, M., and Green, J. C., "The SLICE, CHESS, and SISTINE Ultraviolet Spectrographs: Rocket-Borne Instrumentation Supporting Future Astrophysics Missions," Journal of Astronomical Instrumentation 5, 1640001 (Mar. 2016).

[6] Kruczek, N., Nell, N., France, K., Hoadley, K., Fleming, B., Kane, R., Ulrich, S., Egan, A., and Beatty, D., "The third flight of the Colorado high-resolution echelle stellar spectrograph (CHESS): improvements, calibrations, and preliminary results," in [Society of Photo-Optical Instrumentation Engineers (SPIE) Conference Series], Society of Photo-Optical Instrumentation Engineers (SPIE) Conference Series 10397, 103971G (Aug. 2017). 
[7] Snow, T. P. and Jensen, A. G., "Interstellar Abundances and Depletions Based On FUSE Spectra," in [Astrophysics in the Far Ultraviolet: Five Years of Discovery with FUSE], Sonneborn, G., Moos, H. W., and Andersson, B.-G., eds., Astronomical Society of the Pacific Conference Series 348, 457 (June 2006).

[8] Burgh, E. B., France, K., and McCandliss, S. R., "Direct Measurement of the Ratio of Carbon Monoxide to Molecular Hydrogen in the Diffuse Interstellar Medium," ApJ (Mar. 2007).

[9] Burgh, E. B., France, K., and Jenkins, E. B., "Atomic and Molecular Carbon as a Tracer of Translucent Clouds," ApJ 708, 334-341 (Jan. 2010).

[10] Jenkins, E. B. and Tripp, T. M., "The Distribution of Thermal Pressures in the Interstellar Medium from a Survey of C I Fine-Structure Excitation," ApJS 137, 297-340 (Dec. 2001).

[11] France, K., Nell, N., Kane, R., Burgh, E. B., Beasley, M., and Green, J. C., "H Excitation Structure on the Sightlines to $\delta$ Scorpii and $\zeta$ Ophiuci: First Results from the Sub-orbital Local Interstellar Cloud Experiment," ApJL 772, L9 (July 2013).

[12] France, K., Nell, N., Hoadley, K., Kane, R., Burgh, E. B., Beasley, M., Bushinksy, R., Schultz, T. B., Kaiser, M., Moore, C., Kulow, J., and Green, J. C., "Flight performance and first results from the suborbital local interstellar cloud experiment (SLICE)," in [UV, X-Ray, and Gamma-Ray Space Instrumentation for Astronomy XVIII], Proc. SPIE 8859, 885910 (Sept. 2013).

[13] Kane, R., Nell, N., Schultz, T., France, K., Beasley, M., Burgh, E., Bushinsky, R., and Hoadley, K., "The opto-mechanical design of the sub-orbital local interstellar cloud experiment (SLICE)," in [UV, X-Ray, and Gamma-Ray Space Instrumentation for Astronomy XVIII], Proc. SPIE 8859, 885912 (Sept. 2013).

[14] Hoadley, K., France, K., Kruczek, N., Fleming, B., Nell, N., Kane, R., Swanson, J., Green, J., Erickson, N., and Wilson, J., "The re-flight of the Colorado high-resolution Echelle stellar spectrograph (CHESS): improvements, calibrations, and post-flight results," in [Space Telescopes and Instrumentation 2016: Ultraviolet to Gamma Ray], Proc. SPIE 9905, 99052V (July 2016).

[15] Prinja, R. K., Massa, D., Fullerton, A. W., Howarth, I. D., and Pontefract, M., "Wind variability of B supergiants. II. The two-component stellar wind of $\gamma$ Arae.," A\&\&A 318, 157-170 (Feb. 1997).

[16] Jenkins, E. B., Joseph, C. L., Long, D., Zucchino, P. M., and Carruthers, G. R., "IMAPS - A high-resolution, echelle spectrograph to record far-ultraviolet spectra of stars from sounding rockets," in [Ultraviolet technology II], Huffman, R. E., ed., Proc. SPIE 932, 213-229 (Jan. 1988).

[17] Thomas, R. J., "Toroidal varied-line space (TVLS) gratings," in [Innovative Telescopes and Instrumentation for Solar Astrophysics], Keil, S. L. and Avakyan, S. V., eds., Proc. SPIE 4853, 411-418 (Feb. 2003).

[18] Vallerga, J., Raffanti, R., Tremsin, A., Siegmund, O., McPhate, J., and Varner, G., "Large-format highspatial resolution cross-strip readout MCP detectors for UV astronomy," in [Space Telescopes and Instrumentation 2010: Ultraviolet to Gamma Ray], Proc. SPIE 7732, 773203 (July 2010).

[19] Siegmund, O. H. W., Tremsin, A. S., and Vallerga, J. V., "Development of cross strip MCP detectors for UV and optical instruments," in [UV, X-Ray, and Gamma-Ray Space Instrumentation for Astronomy XVI], Proc. SPIE 7435, 74350L (Aug. 2009).

[20] Landsman, W. and Bowers, C., "Scattered Light in the STIS Echelle Modes," in [The 1997 HST Calibration Workshop with a New Generation of Instruments], Casertano, S., Jedrzejewski, R., Keyes, T., and Stevens, M., eds., 132 (Jan. 1997).

[21] Fleming, B., McCandliss, S., Kaiser, M., Kruk, J., Feldman, P., Kutyrev, A., Li, M., Rapchun, D., Lyness, E., Moseley, S. H., Siegmund, O., Vallerga, J., and Martin, A., "Fabrication and calibration of fortis," (2011).

[22] Beasley, M., Boone, C., Cunningham, N., Green, J., and Wilkinson, E., "Imaging Spectrograph for Interstellar Shocks: A Narrowband Imaging Payload for the Far Ultraviolet," A. Opt. 43, 4633-4642 (Aug. 2004).

[23] Green, J. C., Wilkinson, E., and Morse, J. A., "The Cosmic Origins Spectrograph," in [Future EUV/UV and Visible Space Astrophysics Missions and Instrumentation.], (2003).

[24] Jacquot, B. C., Monacos, S. P., Hoenk, M. E., Greer, F., Jones, T. J., and Nikzad, S., "A system and methodologies for absolute quantum efficiency measurements from the vacuum ultraviolet through the near infrared," Review of Scientific Instruments 82, 043102-043102 (Apr. 2011). 
[25] Nikzad, S., Hoenk, M. E., Grunthaner, P. J., Terhune, R. W., Grunthaner, F. J., Winzenread, R., Fattahi, M. M., Tseng, H.-F., and Lesser, M. P., "Delta-doped CCDs: high QE with long-term stability at UV and visible wavelengths," in [Instrumentation in Astronomy VIII], Crawford, D. L. and Craine, E. R., eds., Proc. SPIE 2198, 907-915 (June 1994).

[26] Nikzad, S., Jewell, A. D., Hoenk, M. E., Jones, T. J., Hennessy, J., Goodsall, T., Carver, A. G., Shapiro, C., Cheng, S. R., Hamden, E. T., Kyne, G., Martin, D. C., Schiminovich, D., Scowen, P., France, K., McCandliss, S., and Lupu, R. E., "High-efficiency UV/optical/NIR detectors for large aperture telescopes and UV explorer missions: development of and field observations with delta-doped arrays," Journal of Astronomical Telescopes, Instruments, and Systems 3, 036002 (July 2017).

[27] Nell, N., France, F., Harwit, A., Bradley, S., Franka, S., Freymiller, E., and Ebbets, D., "Characterization of an ultraviolet imaging detector with high event rate roic (heroic) readout," (2016).

[28] McCandliss, S. R., "Molecular Hydrogen Optical Depth Templates for FUSE Data Analysis," PASP 115, 651-661 (June 2003). 